$\begin{array}{cl}\begin{array}{c}\text { Revue } \\ \text { de } / \text { histoire } \\ \text { des religions }\end{array} & \text { Revue de l'histoire des religions } \\ & \begin{array}{l}\mathbf{4} 2006 \\ \text { Varia }\end{array}\end{array}$
Salomon et le jeune homme à la coupole de verre.
Remarques sur un conte sapiential morisque

Solomon and the young man in the glass alcuba. Notes on a morisco wisdom tale

François Delpech

Édition électronique

URL : http://journals.openedition.org/rhr/5221

DOI : $10.4000 /$ rhr.5221

ISSN : 2105-2573

Éditeur

Armand Colin

Édition imprimée

Date de publication : 1 décembre 2006

Pagination : 439-486

ISBN : 978-2-2009-2106-4

ISSN : 0035-1423

Référence électronique

François Delpech, « Salomon et le jeune homme à la coupole de verre. Remarques sur un conte sapiential morisque », Revue de l'histoire des religions [En ligne], 4 | 2006, mis en ligne le 29 janvier 2010, consulté le 30 avril 2019. URL : http://journals.openedition.org/rhr/5221 ; DOI : 10.4000/ rhr.5221 


\section{Salomon et le jeune homme à la coupole de verre Remarques sur un conte sapiential morisque}

Examen des corrélats mythiques, folkloriques et littéraires d'un conte morisque où sont mis en présence le roi Salomon et un jeune homme vivant en pleine mer dans une coupole de verre. Sont mis en relief les croisements de la légende salomonienne avec plusieurs cycles de récits orientaux où transparaît un diffus héritage indo-iranien.

Solomon and the young man in the glass alcuba.

Notes on a morisco wisdom tale.

Study of the mythical, folkloric and literary affinities of a morisco tale in which a young man living at sea in a glass bowl gets acquainted with king Solomon. Some intersections are underlined between the legend of Solomon and several oriental narrative cycles, variously related to an indo-iranian cultural legacy. 


\section{UNE AVENTURE INSOLITE DU ROI SALOMON}

1) Le curieux récit qui fait l'objet de cette étude peut être à juste titre considéré comme l'un des chefs d'œuvre mineurs de la littérature aljamiada des Morisques de la péninsule Ibérique. On sait que cette littérature, écrite en espagnol avec des caractères arabes, est le bien propre de ces crypto-musulmans qui, en principe convertis au christianisme après la fin de la Reconquista (1492), sont demeurés en Espagne et y ont plus ou moins secrètement continué à pratiquer leur religion, jusqu'à leur expulsion définitive (à la fin de la première décennie du XVII ${ }^{\mathrm{e}}$ siècle) ${ }^{1}$.

Largement acculturés, les Morisques ont perdu nombre de leurs repères : sans encadrement religieux stable, ayant plus ou moins complètement oublié l'arabe, donc l'accès à leurs textes sacrés, ils ont cependant réussi, dans un environnement hostile ${ }^{2}$, à préserver une partie de leur univers mental en se construisant un imaginaire rapiécé. Quoique fondamentalement musulmane, la littérature qu'ils ont cultivée en secret, transmise sous le manteau, cachée dans des faux plafonds, est le produit d'un véritable bricolage culturel : des éléments d'origines multiples, démembrés et reconstruits à grand renfort de contaminations diverses et parfois de falsifications ${ }^{3}$, y ont été intégrés, souvent mêlés à des traditions orales dont on découvre occasionnellement qu'elles nous ont conservé telle croyance ou tel mythe d'origine orientale qu'on avait pu croire oubliés depuis longtemps ${ }^{4}$.

1. Antonio Domínguez Ortiz, Bernard Vincent, Historia de los Moriscos, $2^{\mathrm{e}}$ éd., Madrid, Alianza, 1997 ; Francisco Márquez Villanueva, El problema morisco (desde otras laderas), Madrid, Ed. Libertarias, 1998.

2. Louis Cardaillac, Morisques et Chrétiens, un affrontement polémique (1492-1640), Paris, Klincksieck, 1977.

3. Voir notamment la fameuse affaire des «Livres de plomb » de Grenade (ou « apocryphes du Sacromonte ») qui a fait couler beaucoup d'encre : parmi les travaux les plus récents voir ceux qui ont été réunis dans la revue $A l$ Qantara, XXIII, 2002 et XXIV, 2003.

4. Quelques classiques du conte arabe (connus notamment par les versions littéraires recueillies dans les Mille et une nuits), comme l'histoire de la Ville de cuivre, ou l'aventure de Buluqiyâ, sont ainsi passés dans la littérature morisque : voir Federico Corriente Córdoba, Relatos píos y profanos del ms. aljamiado de Urrea de Jalón, Saragosse : Inst. Fernando el Católico, 1990, p. 227-252, et Luce López Baralt, El Viaje Maravilloso de Buluqiyâ a los confines del universo, Madrid, Trotta, 2004. 
Plusieurs de ces textes nous ont ainsi restitué une histoire sainte revisitée, souvent atypique, farcie de personnages et d'épisodes inédits, dont on ne sait trop s'ils procèdent de quelque ancienne tradition orale perdue ou d'une manipulation tardive du patrimoine légendaire hérité.

Certains personnages bibliques apparaissent dans cette littérature ${ }^{5}$, sous une forme certes islamisée ${ }^{6}$, quoique parfois insolite, et le roi sage par excellence, Salomon, y joue un rôle privilégié 7 . C'est précisément une aventure inédite de Salomon, épisode apparemment inconnu des grands textes qui nous ont rapporté la geste du fils de David, qui fait l'objet du bref récit dont il va être question.

Ce conte figure dans une collection sapientiale inédite intitulée Libro de los Castigos, qui remonte probablement à la fin du $\mathrm{XVI}^{\mathrm{e}}$ siècle ou aux premières années du XVII ${ }^{\mathrm{e} 8}$. Quoique ce récit soit

5. Antonio Vespertino Rodríguez, Leyendas aljamiadas y moriscas sobre personajes bíblicos, Madrid, Gredos (CLEAM 6), 1983.

6. Comme c'est le cas dans l'ensemble de la littérature musulmane : voir D. Sidersky, Les origines des légendes musulmanes dans le Coran et dans les Vies des Prophètes, Paris, Geuthner, 1933 et Roberto Tottoli, Biblical Prophets in the Qu'rân and Muslim Literature, Richmond (Surrey), Curzon, 2002.

7. A. Vespertino Rodríguez, op. cit., p. 42-49 et 247-271. Voir également Joaquina Albarracín Navarro, José Martínez Ruiz, Medicina, farmacopea y magia en el Misceláneo de Salomón (texto árabe, traducción, glosas aljamiadas y glosario), Grenade, Univ. de Grenade, 1987 ; Yvette Cardaillac-Hermosilla, La Magie en Espagne: Morisques et Vieux-Chrétiens aux XVI et XVII siècles, Zaghouan, Fondation Temimi, 1996, p. 133-147. Sur le rôle de la légende salomonienne dans les Apocryphes du Sacromonte voir Philippe Roisse, «L'Histoire du Sceau de Salomon, ou de la Coincidentia Oppositorum dans les "Livres de Plomb" », Al Qantara, XXIV (2), 2003, p. 360-407.

8. María José Fernández Fernández, Libro de los Castigos (ms. aljamiado $n^{\circ} 8$ de la B. de la Junta), Edición, estudio lingüístico, glosario y notas, 3 vol., thèse inédite de l'Université d'Oviedo, 1987. Le ms n 8 de la Biblioteca de la Junta de Estudios Árabes de Madrid a été découvert à Almonacid de la Sierra (Saragosse) : il aurait été rédigé au XVII ${ }^{\mathrm{e}} \mathrm{s}$. et contient notamment le texte de l'histoire de Buluqiyâ : voir Julián Ribera, Miguel Asín Palacios, Manuscritos árabes y aljamiados de la Biblioteca de la Junta, Madrid, Centro de Estudios Históricos, 1912, et L. López Baralt, op. cit., p. 22. Le Libro de los Castigos relève d'un genre sapiential, précocement et largement représenté dans l'ensemble du monde musulman, qui combine proverbes, apophtegmes, anecdotes et récits plus développés ; la littérature morisque a largement cultivé cette veine. Voir Régis Blachère, «Contribution à l'étude de la Littérature Proverbiale des Arabes 
complètement étranger, jusqu'à plus ample informé, aux traditions salomoniennes juives et musulmanes dûment répertoriées ${ }^{9}$, on y reconnaît de nombreux éléments d'origine orientale, et on ne peut lui dénier une cohérence signifiante d'autant plus « logique » qu'elle apparaît ancrée dans un imaginaire qui, s'il revêt ici une forme originale, n'en reconduit pas moins à certaines représentations fondamentales des cultures musulmanes concernant les différents types de sagesse.

Tout se passe comme si le narrateur avait voulu souligner l'éventuelle origine «folklorique » de son histoire : introduite par un simple «Fue recontado que... », cette dernière n'est pas précédée du traditionnel rappel de la chaîne des auteurs qui auraient pu en assurer la transmission ; elle n'est pas davantage encadrée de sentences ou de gloses susceptibles d'en expliciter le sens; elle est construite selon le principe bien connu des récits emboîtés (récit-cadre + récit introduit, chacun de ces deux segments narratifs comportant en outre un petit récit dans le récit, comme il arrive généralement dans les collections du type des Mille et une Nuits); enfin le style en est clairement formulaire, répétitif (multiplication des occurrences de la plus élémentaire des conjonctions de coordination, « $i »$, en guise

à l'époque archaïque », Arabica, VII, 1954, p. 53-83 ; F. Corriente Córdoba, op. cit., p. 35-36 ; Ảlvaro Galmes de Fuentes, « La literatura española aljamiadomorisca », in W. Mettmann ed., Grundriss der Romanischen Literaturen des Mittelalters, vol. IX, La littérature dans la péninsule ibérique aux XIVe et $X V^{e}$ siècles, Heidelberg, C. Winter, 1985, t. 1, p. 117-132 (en particulier p. 128), t. 2, p. 103-112 (voir les n ${ }^{\circ} 4888$, 4890, 4892, 4894, 4896, 4898, 4944, parmi lesquels figurent plusieurs recueils de castigos) ; Ottmar Hegyi, Cinco leyendas y otros relatos moriscos (ms. 4953 de la Biblioteca Nacional de Madrid), Madrid, Gredos, CLEAM n 4, 1981, p. 99-104, 131-135. Sur le rôle des éléments narratifs dans cette littérature prioritairement didactique voir Manuela Manzanares de Cirre, «El capítulo de las racontaciones del libro del Samarkandi », in Actas del Coloquio Internacional sobre literatura aljamiada y morisca, Madrid, Gredos, 1978, p. 237-258.

9. Voir Louis Ginzberg, Les légendes des Juifs, trad. fr., t. V, Paris, Cerf, 2004, p. 91-127, 226-251, et l'article « Sulaymân b. Dâwûd » de l'Encyclopédie de l'Islam ( $2^{\mathrm{e}}$ éd.), t. IX, 1998, p. 857 sq. Pour une collection riche, commode et bibliographiquement bien documentée de légendes salomoniennes, voir John D. Seymour, Tales of King Solomon, Londres, Oxford Univ. Pr., 1924. 
d'articulation des micro-séquences), comme c'est le cas dans les récits qui relèvent de la tradition orale.

2) Tout commence par l'évocation de l'accès de curiosité cosmique qui induit Salomon à sélectionner (par écrémage progressif) trois démons ( «assaytanes ») afin de les envoyer explorer la mer, avec la mission de découvrir quels en sont les habitants et d'en sonder la profondeur ${ }^{10}$.

Le premier démon-plongeur, plus particulièrement chargé d'examiner les occupations des créatures marines, visite toutes les mers, n'y rencontre que des poissons et se rend compte qu'y règne l'équivalent maritime de la loi de la jungle : les plus gros dévorent les plus petits ${ }^{11}$. Il revient alors rendre compte à Salomon du résultat de cette première exploration.

Le second démon, parti en quête du fond de la mer, ne parvient pas à accomplir sa mission : le fond s'avère inaccessible. Il rencontre d'ailleurs un ange («almalaque») qui, premier récit intercalé, lui raconte que, cent ans auparavant, il a vu un marin laisser échapper par dessus bord un outil avec lequel il effectuait une réparation sur son bateau : l'outil en question n'a toujours pas atteint le fond! Seul Allah connaît la profondeur de l'abîme... Le démon poursuit néanmoins son investigation sous-marine et finit par découvrir un objet inattendu, à savoir une «alcuba » de verre verte, remuée par les flots et combattue par les poissons, qui ne parviennent cependant pas à lui causer le moindre dommage ${ }^{12}$. Il revient faire part à Salomon

10. Le conte est reproduit (p. $226 s q$.), partiellement traduit (p. $227 s q$.) et brièvement commenté (p. 138 sq.) par Y. Cardaillac Hermosilla, op. cit., que je remercie de m'avoir aimablement communiqué un exemplaire de l'édition de sa thèse.

11. On sait que dans les textes sanscrits la « loi de la jungle » est généralement appelée «loi des poissons».

12. Y. Cardaillac traduit alcuba par «flacon». Le contexte semble bien impliquer en effet qu'il s'agit d'une sorte de récipient de verre clos, bocal, tonneau ou cuve. Il faut cependant noter (je remercie A. Chraïbi de me l'avoir fait remarquer) que nous avons ici affaire, sous une forme hispanisée, au mot arabe signifiant «coupole» $(a l-K u b b a)$. Cet élément architectural n'étant généralement pas en verre, et encore moins destiné à jouer le rôle de résidence sous-marine mobile, il semble bien que le narrateur morisque, qui a dû transposer un récit arabe (c'est-à-dire composé originellement dans une langue qu'il 
de sa découverte. Le roi envoie alors ses cent démons les plus compétents ${ }^{13}$, qu'il charge de lui ramener le mystérieux objet.

L'opération ayant été menée à bien sans délai, et la cuve de verre étant présentée à Salomon, voici qu'en sort un jeune homme («mancebo ») vêtu de blanc ${ }^{14}$, lequel, à la demande du roi, commence aussitôt à raconter son histoire (deuxième récit dans le récit).

Il avait vécu avec ses parents : ceux-ci ayant atteint l'extrême vieillesse, il s'était occupé d'eux, les lavant et les nourrissant comme on le fait pour de très jeunes enfants. Après la mort de son père il était resté avec sa mère et avait continué de même. Au moment de

ne dominait plus), a interprété le mot à la lumière de son homophone castillan cuba (« tonneau, cuve ») qui lui était certainement plus familier. Cette « traduction » spontanée a pu déterminer une modification du contenu même du récit. Noter que le même mot $(a l-q u b b a)$, cette fois avec son sens originel, figure dans l'une des versions aljamiadas du roman d'Alexandre : voir Alois Richard Nykl, «Aljamiado Literature. El Rrekontamiento del rey Alisandre », Revue Hispanique, LXVII, 1929, p. 409-611 (p. 547). Voir infra n. 14 et n. 52.

13. On remarque qu'il n'est plus question du dernier des trois démons initialement sélectionnés par Salomon: Y. Cardaillac (op. cit., p. 138) voit dans cet « oubli » un procédé littéraire visant à ménager un certain « suspense ». On peut cependant se demander si ce n'est pas, comme la réinterprétation du mot alcuba, l'indice d'un rifacimento hâtif et condensé d'un original plus complexe. Voir infra nn. 47 et 69.

14. Ce n'est qu'à la fin du conte que l'on apprend que la cuve de verre est munie d'une porte, qui peut se refermer de l'intérieur : il semble donc bien qu'il s'agit d'un habitacle clos. Sa situation exacte au sein de la mer n'est pas davantage précisée : flotte-t-elle à la surface de l'eau (comme pourrait le faire une coupe, semblable à celle d'Helios) - c'est ce que suppose la traduction d'Y. Cardaillac - ou a-t-elle été trouvée en profondeur par le démon-plongeur ? Est-elle implantée sur quelque îlot rocheux (comme pourrait l'être une coupole) ? Bien qu'elle soit quotidiennement visitée par un oiseau (ce qui pourrait laisser penser qu'elle flotte à l'air libre ; mais on peut aussi imaginer qu'il s'agit d'un oiseau plongeur), le fait qu'elle soit « combattue » par les poissons et qu'il ne soit pas possible d'y voir la lumière du jour induit l'hypothèse d'un objet sous-marin. Enfin l'allusion aux transferts dont elle fait l'objet parait écarter l'idée d'une architecture fixe implantée sur quelque support immobile (le fond étant de toute façon à exclure puisqu'on sait qu'il est inaccessible). L'existence de la porte autorise l'hypothèse d'une sorte de bathyscaphe, susceptible de se déplacer entre la surface et les profondeurs : $c f$. infra $\mathrm{n} .84$. 
mourir à son tour, sa mère avait prié Allah de mettre son fils à l'abri des vices et des tentations du monde, afin de lui préserver le capital de mérites («aquel gualardón») qu'il s'était acquis par sa dévotion filiale. Ainsi fut fait : après la mort de sa mère, deux anges vinrent s'emparer de lui, l'emmenèrent et le déposèrent dans la cuve où le démon l'a trouvé.

Après ce bref curriculum s'ensuivent une série de questions et de réponses, au cours desquelles Salomon apprend du jeune homme qu'il y a vingt-six ans qu'il se trouve ainsi enfermé dans son habitacle de verre, qu'il est quotidiennement visité par un oiseau blanc qui lui apporte, en guise de nourriture, une substance blanche qui suffit à le rassasier et désaltérer, et qu'il ne distingue le jour et la nuit que grâce à deux veines ou rainures ${ }^{15}$, dont l'une, qui est blanche, grandit quand il fait jour, tandis que l'autre, noire, s'accroît quand il fait nuit.

Au terme de cet échange, Salomon demande au jeune homme s'il veut bien rester avec lui. Mais le garçon refuse et demande à être remis dans sa situation initiale, afin que s'accomplisse le jugement d'Allah. Il rentre donc dans son habitacle, en referme la porte de l'intérieur, et les démons le reconduisent en mer pour le déposer à l'endroit où ils l'avaient trouvé.

3) Quoique non explicite et relativement complexe, le contenu sapiential de ce conte est assez clair pour que le lecteur (ou l'auditeur) n'ait pas besoin d'explication complémentaire : il lui suffit, pour en saisir les trois niveaux de sens inextricablement entremêlés - à savoir la « leçon de choses » sur la nature de l'univers et de la société, le message moral et ascétique, et l'ouverture finale sur une forme de spiritualité proche d'une mystique de l'abandon bienheureux - de suivre palier après palier les étapes du cheminement intellectuel, pour ainsi dire initiatique, qu'imposent à Salomon les résultats inattendus de l'expérience inédite dont il a lui-même, quelque peu inconsidérément, pris l'initiative.

15. Ici encore l'imprécision règne : faut-il supposer que ces vetas sont situées dans la paroi du bocal de verre ? On verra que le même motif intervient dans d'autres textes sous une forme très différente. Voir infra n. 101. 
Cet enchaînement de découvertes successives, chacune plus stupéfiante que la précédente, tout à la fois hautement symboliques et matérialisées dans des images très concrètes, amène en effet le Maître de Sagesse à prendre à son tour, pour une fois, une bonne leçon. Une leçon finalement plutôt humiliante pour lui...

Il apprend d'abord de quelle transgressive vanité il a fait preuve en cédant à sa curiosité cosmologique : l'univers (la mer) est insondable et seul Allah en connaît le dernier mot.

Il découvre ensuite que la vie des hommes, manifestement symbolisée par les mœurs des poissons, est exclusivement dominée par les rapports de force (lesquels ne sont même pas ici tempérés par le sage recours à la ruse et à la prudence, que préconisent tant d'apologues sapientiaux et autres « miroirs des princes »).

Il comprend surtout que la vraie sagesse réside dans cet obscur dévouement filial - qui est le seul remède à l'état d'enfantine dépendance auquel le passage du temps réduit inévitablement les hommes - et dans un retrait final et absolu du monde, qui seul permet de préserver des mérites si chèrement et humblement conquis.

Il doit en outre reconnaître que cette morale, fondée sur la réciprocité et le secret, est plus que toute autre agréée et récompensée par Allah : alors que lui, Salomon, ne commande qu'à des démons, d'ailleurs incompétents (eu égard aux tâches qu'il leur assigne), le jeune homme encapsulé est, pour sa part, miraculeusement servi par des anges, sans avoir même à leur commander quoi que ce soit.

Subissant enfin l'humiliation du refus opposé par le jeune renonçant à son offre de le garder avec lui, Salomon est donc mis en présence d'une sagesse incommensurable à la sienne.

Implicitement obligé d'admettre la vanité des grandeurs mondaines (et royales), la relativité de son savoir et les limites de son pouvoir, amené à constater qu'il n'a pas l'exclusivité (ni même la meilleure part) des faveurs divines, Salomon assiste en somme à la remise en perspective du type de Sagesse dont il est, aux yeux du monde, la plus haute et notoire incarnation.

Ce rééquilibrage aboutit en effet à l'exaltation, discrète mais efficace, d'un renoncement au monde qui ne ressemble qu'extérieurement à 
une anachorèse ascétique ${ }^{16}$, et apparaît comme le résultat quasi automatique d'un mécanisme cyclique fondé sur la réciprocité du don et la récurrence alternative d'un état d'enfance : celui qui, dans son enfance, a été soigné, lavé et nourri par ses parents, soignera, lavera et nourrira à son tour ces derniers lorsque l'âge les aura ramenés à l'état d'enfance ; une fois ses parents disparus, il sera lui-même à nouveau pris en charge, encapsulé entre mer et ciel (et comme réinstallé dans ce substitut utérin qu'est le bocal où il va se trouver reclus), protégé et nourri par une instance supérieure qui le maintiendra, vrai puer senex ${ }^{17}$, dans un état d'inaltérable et passive absconditio, immunisé comme les Sept Dormants contre les ravages du Temps, la souillure et la violente loi du Monde ${ }^{18}$.

Ce récit fait a priori figure de hapax : l'absence de source manifeste ou de parallèle dans les traditions salomoniennes juives et musulmanes les plus répandues pourrait laisser penser qu'il s'agit d'un artefact purement morisque, d'un patchwork fabriqué à partir de motifs légendaires hétérogènes. Le procédé consistant à entremêler dans un même récit des éléments issus d'histoires différentes ${ }^{19}$, ou à attribuer à un personnage des aventures originellement associées

16. La solitude cosmique où se trouve plongé notre jeune renonçant n'est pas le fait d'une décision hérö̈que, ni même d'un parti pris délibéré : elle est comme le prolongement naturel d'une vie discrètement consacrée à la plus élémentaire des dévotions ; le miracle dont il a bénéficié ne résulte même pas d'un vœu qu'il aurait formulé personnellement, mais d'une prière de sa mère, dont il subit passivement la conséquence. Son absorption inerte dans un état presque indifférencié, où le jour ne se distingue pas de la nuit, apparaît donc comme une sorte de retour consenti à l'état prénatal.

17. Notre mancebo ne peut être tel que par l'effet d'une suspension miraculeuse du temps : s'il était soumis à celui des horloges il ne serait plus le jeune homme qu'affirme le récit puisqu'il a assisté ses parents jusqu'à la fin de leur extrême vieillesse avant de passer, par la suite, vingt-six années supplémentaires enfermé dans son bocal... !

18. Sur le mystère des Sept Dormants de la Caverne dans le monde musulman voir le travail classique de Louis Massignon, « Les Sept Dormants, apocalypse de l'Islam », Analecta Bollandiana, LXVIII, 1950, p. 245-260, ainsi que François Jourdan, La tradition des Sept Dormants. Une rencontre entre Chrétiens et Musulmans, Paris, Maisonneuve et Larose, 1983, et Julia Hernández Juberías, La Península Imaginaria. Mitos y leyendas sobre Al-Andalus, Madrid, CSIC, 1996, p. 121-161.

19. M. Manzanares de Cirre, op. cit., p. 243 sq. 
à un autre ${ }^{20}$, pourrait en effet passer pour une caractéristique de la littérature aljamiada si on ne le trouvait déjà dans certains des modèles arabes qu'elle transpose ou dont elle s'inspire ${ }^{21}$. Il faut toutefois remarquer que s'il y a eu contamination ou remontage, le résultat n'en reste pas moins cohérent et bien construit.

Une autre hypothèse serait d'ailleurs envisageable : nous pourrions nous trouver en face d'une relique, ou d'une résurgence d'une tradition orale perdue, que, pour une raison indéterminée, les Morisques auraient été les seuls à préserver et transmettre.

On ne saurait, en tout état de cause, formuler de conjecture à ce sujet avant d'avoir au moins esquissé l'analyse narratologique des composantes du récit et de leurs éventuelles coordonnées dans les cultures dont les Morisques étaient tributaires.

\section{CONTES SAPIENTIAUX ET LÉGENDES DE THÉODICÉE}

1) Le thème général du conte, c'est-à-dire la mise en présence contrastive de Salomon et d'un personnage mystérieux, avec lequel il aura un échange (potentiellement) sapiential - lequel ne tournera pas nécessairement à l'avantage du roi - rappelle évidemment toute la tradition des débats ou concours de « sagesse ${ }^{22}$ salomoniens.

20. A. Vespertino Rodríguez, op. cit., p. 46 sq. et 247 sq. (invention d'une nouvelle aventure de Salomon moyennant une contamination avec l'histoire de Daniel et la Chaste Suzanne).

21. Remarquer les analogies multiples entre notre conte et le Capítulo de las Racontaciones de l'Advenimiento de los descuidados, traduction aljamiada du Tambih al-Gafilin d'al-Samarqandi (M. Manzanares de Cirre, op. cit.), où l'on trouve des récits sur les thèmes du renoncement aux richesses (ibid., p. 253 sq.), du suicide destiné à fuir une tentation susceptible de faire perdre les mérites antérieurement acquis (ibid., p. $246 s q$. : on retrouve dans ce conte les motifs de l'ange salvateur et de la nourriture miraculeuse), de l'opposition entre la sphère royale et la spiritualité ascétique (p. 249 sq. : l'histoire du fils d'Harun al Rashid, qui refuse d'obéir à son père et dont le parti-pris ascétique est récompensé par la visite d'un oiseau merveilleux), du miracle déterminé par la piété filiale (p. 246), de la puissance magique de la bénédiction (ou malédiction) maternelle (p. 254 sq.).

22. Il s'agit en fait le plus souvent d'intelligence et d'astuce. Ces échanges tendent tantôt à mettre en évidence la supériorité de la « sagesse » de Salomon, tantôt à la relativiser, voire à la ridiculiser en la parodiant. 
Ces échanges, souvent dialogués, prennent généralement la forme de concours d'énigmes, de questions et de réponses, d'aphorismes croisés.

L'interlocuteur du roi sage y est le plus souvent un personnage radicalement « autre », parfois un antagoniste plus ou moins déclaré. Qu'il s'agisse de la reine de Saba, du roi phénicien Hiram, de l'énigmatique Abdemon, du démon Asmodée (ou de son alter ego arabe Shakr), du «centaure » Kitovras, des anges déchus Azza et Azzael, ou, dans les textes médiévaux, de l'étrange et païen « fou-sage » dénommé Marcolf ou Saturne ${ }^{23}$, cet interlocuteur allogène est souvent associé à l'eau (ou plus particulièrement à la mer) ${ }^{24}$, parfois au verre (c'est le cas dans la légende de l'obtention du shamir, dont on sait que, sur la recommandation d'Asmodée, c'est en recouvrant d'un couvercle de verre le nid de l'oiseau qui le détient que l'envoyé de Salomon parvient à s'en emparer) ${ }^{25}$, et il arrive qu'il soit à l'origine d'une humiliation infligée à Salomon ${ }^{26}$.

Il faut reconnaître cependant que, malgré ces analogies circonstancielles, le conte morisque ne se rattache que de loin à ce cycle de récits sapientiaux : il y a bien un échange de questions et de réponses entre Salomon et le jeune homme vêtu de blanc, mais elles sont à sens unique et il n'y a pas de débat entre les deux interlocuteurs, ni d'évaluation comparative de leurs intelligences et savoirs respectifs. À l'inverse, le motif si spécifique du jeune homme enfermé dans un habitacle de verre, qui constitue la donnée la plus originale et distinctive du conte, ne se trouve pas dans la tradition des concours de sagesse salomoniens ${ }^{27}$.

23. Voir Robert J. Menner, The poetical dialogues of Solomon and Saturn, New York, Yale Univ., 1941, p. 22 sq.

24. R. J. Menner, "The Vasa Mortis passage in the Old English Solomon and Saturn", in Mélanges F. Klaeber, Minneapolis, 1929, p. 248-249.

25. L. Ginzberg, op. cit., t. V, p. 121 sq.

26. Le cas le plus flagrant est bien sûr celui de la "pénitence» (perte provisoire de son identité et de son royaume) subie par Salomon à la suite du vol de son anneau par Asmodée (ibid., p. 122-124). D'autres légendes moins connues mettent Salomon en difficulté pour avoir présumé de son savoir ou de son pouvoir : J. D. Seymour, op. cit., p. 48-55, 70-72 et passim.

27. Voir infra l'examen des possibles relations de notre conte avec le thème des démons embouteillés. 
Par contre, le récit morisque s'apparente clairement à d'autres traditions sapientiales, où sont conjointement mises en scène une valorisation spécifique de la dévotion filiale et une comparaison relativisante de deux types de sagesse de natures très différentes, l'avantage étant finalement concédé, plus ou moins explicitement, à celui qui a opté pour une pratique discrète (voire secrète), tandis que le sage « professionnel » voit son orgueil rabaissé et sa primauté contestée.

Tel est bien en effet le message du conte morisque : son implicite «morale» revient à une relativisation de la sagesse salomonienne par sa confrontation à une sagesse radicalement autre, toute humaine et terre à terre, dont la pierre de touche est précisément cette humble dévotion filiale confinant à la sainteté, vertu qu'Allah finalement récompense par des faveurs auxquelles le Sage institué qu'est le roi hébreu ne saurait prétendre.

2) Ce schéma idéologique et narratif correspond à un type de conte sapiential manifestement très ancien, d'origine orientale, et largement diffusé, dont le repérage et la définition narratologiques laissent cependant à désirer car les comparatistes qui l'ont étudié ${ }^{28}$ et les folkloristes qui ont mis au point les nomenclatures de référence 29 ont apparemment travaillé séparément, voire sans se connaître.

28. Gordon Hall Gerould, "The Hermit and the Saint", Publications of the Modern Language Association, XX, 1905, p. 529-545 ; Ramón Menéndez Pidal, Estudios Literarios, 7 e éd., Buenos Aires, Espasa Calpe, 1952, p. 13-85, Bernard Heller, «La légende judéo-chrétienne du Compagnon au Paradis », Revue des Etudes Juives, LVI, 1908, p. 198-221. Ce conte-type est tantôt désigné sous le titre « Comparaison des mérites », tantôt sous celui du « Compagnon au Paradis » (ou encore «L'Ermite et le Saint »), et il est souvent mal distingué du cycle «L'Ange et l'Ermite », qui correspond à une catégorie de récit voisine mais différente, le type Aarne-Thompson 759, «God's justice vindicated (the Angel and the Hermit) ».

29. Antti Aarne, Stith Thompson, The types of the folktale, $3^{\mathrm{e}}$ éd., Helsinki, Acad. Sc. Fennica, FFC 184, 1973, p. 260-263. Notre conte y correspond au type 756 D*, « More devout », mais n'y est pas clairement défini et documenté. Ces lacunes et imprécisions nomenclaturales viennent en partie de ce que plusieurs motifs et séquences narratives circulent d'un type de conte à l'autre (c'est le cas pour le thème du saint qui veut savoir qui sera son compagnon au Paradis) à l'intérieur d'un vaste cycle de récits édifiants sur le thème de la théodicée, dont les formes multiples interagissent : voir Haim Schwarzbaum, "The Jewish and Moslem versions of some Theodicy Legends", Fabula, III, 1960, p. 119-169 et, pour les versions arabes, le catalogue de Hasan El-Shamy, Types of the folktale in the Arab World: a demographically oriented Tale-Type Index, Bloomington-Indianapolis, Indiana Univ. Pr., 2004 (types 756 D et 759). 
Dans ces récits, dont la version la plus ancienne se trouve dans le Mahabharata ${ }^{30}$, et dont il existe notamment des versions juives, arabes et espagnoles, un illustre "professionnel de la sagesse » (brahmane, prophète, anachorète, ermite, etc.) que la notoriété de sa dévotion a rendu quelque peu imbu de lui-même, finit par découvrir, à sa grande confusion, qu'un personnage beaucoup moins reluisant que lui ${ }^{31}$ est en fait son égal, ou même son supérieur en sagesse et en mérite, pour la simple raison qu'il assume, avec la dévotion la plus obstinée et la plus discrète, le service de ses parents âgés et impotents.

Le parallélisme est remarquable entre la version indienne ${ }^{32}$ et la version aljamiada (il existe en effet une version morisque de ce conte,

30. Il s'agit de l'histoire du brahmane Kausika et du chasseur, racontée par Mârkandeya à Yudhistira dans le Vana-Parva: voir Johannes Adrianus Bernardus van Buitenen, trad., The Mahabharata, t. II, vol. 3, The Book of the Forest, Chicago-Londres, Univ. of Chicago Pr., 1975, p. 614-638, et Madeleine Biardeau, Le Mahabharata, Paris, Seuil, 2002, t. I, p. 621-644.

31. Il s'agit en effet d'un personnage éminemment lié à la souillure et à la mort (chasseur rencontré dans un abattoir, boucher, etc.). C'est dans le contexte indien, où la classification socio-rituelle statutaire est étroitement liée au type d'activité professionnelle exercé et au degré de pureté correspondant, que le conte prend tout son sens et l'opposition des deux protagonistes toute son acuité. L'implicite critique du brahmanisme que suppose cette opposition laisse peut-être entrevoir une lointaine ascendance bouddhiste de ce genre de récit-débat. Dans plusieurs versions occidentales l'opposition est reportée sur un ermite et un voleur (éventuellement assassin), dont les statuts spirituels initiaux s'inversent in fine car l'ermite se damne par son incompréhension désespérée des voies cachées et apparemment paradoxales de la Justice Divine, qui font que le criminel, repenti in extremis, mérite et obtient en définitive le Salut (voir les types Aarne - Thompson 756 A, «The Self-Righteous Hermit », 756 B, «The Devil's Contract » et 756 C, « The Great Sinner »). Il arrive aussi, cependant, que l'ermite s'amende, accepte la leçon, reconnaisse ses torts et s'humilie, d'où un dénouement plus optimiste. Voir Daniel Devoto, « Don Juan Manuel y "El Condenado por desconfiado" », Bulletin Hispanique, LXVIII, 1966, p. 195-202, qui montre à la fois la solidarité globale et les clivages internes caractéristiques de ce groupe de récits. Voir également Rameline E. Marsan, Itinéraire espagnol du conte médiéval (VIII -XVe s.), Paris, Klincksieck, 1974, p. 213-222.

32. Une autre version indienne se trouve dans le Çukasaptati : voir Theodor Benfey, «Zum guten Gerhard », Germania, XII, 1867, p. 310-318 (p. 316-318). Le brahmane commence par s'indigner des basses occupations du chasseur qu'on lui a présenté comme un parangon de sagesse, mais il doit finalement reconnaître que tel est bien le cas et que lui-même a démérité lorsqu'il a abandonné ses parents pour se livrer à des austérités qui l'ont rendu présomptueux. 
qui se distingue par sa trame narrative de notre récit salomonien, mais s'en rapproche dans la mesure où elle transpose sur le couple Moïse-boucher le contraste sapiential entre Salomon et le jeune homme encapsulé) $)^{33}$ puisque, dans les deux cas, pourtant aussi éloignés dans l'espace que dans le temps, le supersage en titre est déchu de sa suprématie au profit d'un personnage, statutairement suspect ou socialement décrié, associé au trafic de viandes (mais racheté au centuple par la dévotion filiale qu'il pratique plus ou moins secrètement) ${ }^{34}$.

Dans ce groupe de contes le paradoxe sapiential est donc systématiquement cultivé, au profit d'un recadrage fondé sur une mise en cause des apparences et des statuts socialement admis : le sage en titre trouve son maître ou son égal en la personne d'un semiparia exerçant un vil métier, dont l'unique vertu, pratiquée à l'insu de tous, suffit à assurer la suprématie spirituelle.

Le paradoxe est certes moins explicite dans notre conte salomonien : l'humiliation du roi-prophète reste sous-entendue ; mais c'est bien la même humble et obscure dévotion filiale qui est mise en valeur, et opposée à la vaine prétention à la souveraineté cosmique

33. Il s'agit de la légende de Moïse et du boucher Jacob (Alhadiz de Mûçâ kon Yakûb el karniçero) : voir A. Vespertino Rodríguez, op. cit., p. 37-39 et 235-239. Moïse découvre que le boucher Jacob, dont il lui a été révélé qu'il serait son compagnon au Paradis, est un personnage méprisé et de très mauvaise réputation, mais qu'il pratique en cachette la dévotion filiale la plus extrême. Sur ce conte voir René Basset, Mille et un contes, récits et légendes arabes (ed. Aboubakr Chraïbi, Paris, J. Corti, 2005, t. 2, p. 242-244, «Dévouement filial »).

34. Pour un récit juif analogue (contes de la collection de R. Nissim) voir B. Heller, op. cit., p. 198 sq. (cf. ibid., p. 206 sq.), où le vrai sage est également un boucher : l'auteur ignore manifestement les textes indiens et attribue des origines juives à tout le cycle. Pour les versions folkloriques espagnoles modernes, voir R. Menéndez Pidal, op. cit., p. 75-85 : le dévouement du sage plus sage que le sage y atteint une forme héroïque et paradoxale puisque la personne dont il prend soin en secret n'est plus l'un de ses parents mais l'assassin (en fuite) de son père, qu'il cache et entretient avec une abnégation à la fois sublime et absurde ( $c f$. Maxime Chevalier, Cuentos folklóricos españoles del Siglo de Oro, Barcelone, Crítica, 1983, p. 80) : curieusement Julio Camarena et Maxime Chevalier ont ignoré ce type de récit dans leur Catálogo Tipológico del Cuento Folklórico Español. Cuentos Religiosos, Alcalá, Centro de Estudios Cervantinos, 2003. 
que manifeste Salomon en voulant pénétrer les inconnaissables secrets de l'univers.

Salomon se trouve donc, vis-à-vis du jeune homme, embouteillé dans une situation analogue à celle de Moïse (qui a, lui aussi, fait preuve d'une indiscrète et présomptueuse curiosité, à l'égard de la justice divine, lorsqu'il a voulu savoir qui serait son compagnon au Paradis) vis-à-vis du boucher Jacob dans le conte morisque correspondant : les deux leçons sont parallèles, et ce n'est sans doute pas un hasard si, dans les deux cas, c'est un des grands prophètes pré-islamiques qui en fait les frais.

3) Les notations paradisiaques qui sous-tendent ou accompagnent ces édifiantes expériences sapientiales ${ }^{35}$ et la forme délibérément paradoxale affectée par les relations qui en sont faites (qui prennent à rebrousse-poil les hiérarchies instituées) laissent entrevoir que ces récits de théodicée et de comparaison des sagesses sont souterrainement reliés à ce savoir énigmatique que, dans la «mythologie musulmane », représente et incarne le personnage d'al-Khadir ${ }^{36}$.

Le conte morisque de Moïse et du boucher Jacob reprend et adapte en effet le thème initial d'une autre légende, que plusieurs textes musulmans associent à Moïse, où l'on voit également le prophète d'Israel, qui s'est inconsidérément vanté de sa sagesse, recevoir une révélation qui l'amène à entrer en contact avec un mystérieux «serviteur de Dieu» (qui n'est autre qu'al-Khadir), dont les inscrutables actions dépassent l'entendement, somme toute limité, du présomptueux prophète, et dénotent une connaissance supérieure des desseins cachés de Dieu ${ }^{37}$. On sait que cette légende a été adaptée par Mahomet dans le Coran et qu'elle est à l'origine d'un imposant cycle de contes et de récits édifiants qui ont largement

35. Cf. infra n. 108.

36. Voir Arent Jan Wensinck, «al-Khadir», Encyclopédie de l'Islam, $2^{e}$ éd., vol. IV, p. 935-938, Hassan Elboudrari, «Entre le symbolique et l'historique : Khadir immémorial », Studia Islamica, LXXVI, 1992, p. 25-39.

37. A. J. Wensinck, op. cit., p. 936, Migne, Dictionnaire des Apocryphes (Encyclopédie Théologique, t. XXIV), Paris, 1858, t. II, col. 627 sq. ; H. Schwarzbaum, op. cit., p. 149-158. 
circulé dans les cultures concernées par les trois religions du Livre $^{38}$.

La figure de Moïse semble donc avoir joué, au moins dans l'imaginaire morisque, le rôle de pivot autour duquel se sont articulés deux types de conte distincts mais apparentés : celui de la « comparaison des sagesses » (ou du « compagnon au Paradis »), Aa.-Th. $756 \mathrm{D}^{*}$, où le prophète trouve son égal dans la personne d'un homme exerçant un humble et vil métier, et celui de "l'Ange et l'Ermite », Aa.-Th. 759, où il voyage en compagnie d'un personnage surnaturel associé à l'eau ${ }^{39}$, dont les actes paraissent $a$ priori incompréhensibles. Dans les deux cas le prophète de l'Exode est déconfit et édifié, remis à sa place par l'existence ou le comportement de ce compagnon paradoxal et inattendu, et il prend conscience de l'incommensurabilité et/ou du caractère caché de la Justice Divine.

4) Le conte qui fait l'objet de cette étude me semble supposer un report sur le personnage de Salomon d'une partie de cette dualité

38. Aarne-Thompson 759, «God's Justice vindicated (the Angel and the Hermit) », H. Schwarzbaum, op. cit., Id., «Engel und Eremit (Aa-Th. 759)», in Enzyklopädie des Marchens, K. Ranke ed., Berlin-New York, W. de Gruyter, t. III, 1981, col. 1438-1446. Voir également Israel Levi, «La légende de l'Ange et l'Ermite dans les écrits juifs », Revue des Etudes Juives, VIII, 1884, p. 64-73, 202-205 ; Gaston Paris, «L'Ange et l'Ermite. Étude sur une légende religieuse », in La poésie du Moyen Age, Paris, 1885, p. 151-187, Frederic C. Tubach, Index Exemplorum: a handbook of medieval religious tales, Helsinki, Acad. Sc. Fennica, FFC 204, 1969, n 2558 . Pour les versions ibériques voir J. Camarena, M. Chevalier, op. cit., p. 108-117, et María Jesús Lacarra, Cuento y Novela Corta en España. I. Edad Media, Barcelone, Crítica, 1992, p. $278-280$.

39. Voir Israel Friedlaender, « Alexanders Zug nach dem Lebensquell und die Chadhirlegende », Archiv für Religionswissenschaft, XIII, 1910, p. 161-246. Voir également H. T. Norris, Saharan myth and saga, Oxford, Clarendon Pr., 1972, p. 14-21 ; Frederick William Hasluck, Christianity and Islam under the Sultans, Oxford, 1929, t. I, p. 319-336 («El Khidr in the popular religion of Turkey »), et Ananda K. Coomaraswamy, "Khwâjâ Khadir and the fountain of life in the tradition of Persian and Mughal Art", Ars Islamica, I (2), 1934, p. 173-182. Il y aurait lieu d'étudier en détail les rapports entre la légende de Khadir, les mythes grecs des «Vieux de la Mer » et le cycle indo-européen des «Descendants des Eaux» : sur ces deux derniers dossiers voir Dominique Briquel, "Vieux de la mer grecs et Descendant des Eaux indo-européen », in D'Héraklès à Poseidon. Mythologie et Protohistoire, R. Bloch éd., ParisGenève, 1985, p. 141-158. 
contextuelle : d'une part ce récit s'apparente manifestement au cycle narratif fondé sur le thème de la «comparaison des sagesses » (dont il reprend la caractéristique mise en valeur d'une mystique de la discrétion et de la dévotion filiale) ; mais, en faisant de l'interlocuteur de Salomon non plus un humain ordinaire mais un personnage énigmatique, semi-surnaturel, vivant hors du monde et du temps dans une sorte d'éternité aquatique ou de proximité divine, il apparait d'autre part comme relevant de la symbolique khadirienne qui préside au cycle de «l'Ange et l'Ermite ».

Les ambivalences symboliques, superpositions de figures et contaminations de récits hétérogènes sont, on l'a vu, une des caractéristiques de la littérature morisque ${ }^{40}$. Ces recoupements ne se font cependant pas au hasard: ils démultiplient et approfondissent le sens en en diversifiant les points d'appui et en suscitant ou suggérant des analogies.

Notre conte ne saurait certes être considéré comme une version parmi d'autres de «l'Ange et l'Ermite ( et il est, tout aussi bien, une forme assurément aberrante, tout à fait sui generis, du type «Comparaison des sagesses »). La perplexité de Salomon face à l'insondabilité des mers et au mystère du jeune homme à la cuve de verre apparaît bien, néanmoins, au moins implicitement, comme une variante de la stupeur qu'inspirent à Moïse les actions paradoxales de l'énigmatique «Serviteur de Dieu » de la légende coranique, autre traduction de l'inscrutabilité des jugements divins.

De même, $\mathrm{Al}$ Khadir semble a priori inconnu du récit qui nous occupe, où il n'est ni nommé ni évoqué. Loin d'être un étranger pour l'imaginaire morisque ${ }^{41}$, n'est-il pas cependant indirectement présent dans la couleur verte attribuée à la cuve de verre habitée par le mancebo $^{42}$ ?

40. Cf. supra n. 20.

41. Al-Khadir figure bien, tout comme dans leurs modèles arabes, dans les versions aljamiadas de l'histoire de Buluqiyâ (L. López Baralt, op. cit.) et du conte de la Ville de Cuivre (F. Corriente Córdoba, op. cit., p. 250 sq.).

42. On sait que le nom de ce mystérieux «prophète » signifie «le vert» ou « le verdoyant » (voir A. J. Wensinck, op. cit.). 
Tout le contexte maritime du conte n'est-il pas enfin surdéterminé par les affinités prioritairement aquatiques de la mythologie khadirienne ? Ce sont ces connotations latentes, voire subliminales, qu'on essaiera de tirer au clair en examinant les paramètres narratologiques de quelques-uns des principaux motifs «folkloriques » mobilisés par le récit.

\section{LES DÉMONS PLONGEURS ET L'IMAGINAIRE COSMOGONIQUE}

1) Le premier de ces motifs, qui est aussi l'un des plus rares et des plus remarquables, est celui de l'envoi par Salomon d'une série de démons plongeurs chargés d'examiner les fonds marins.

Ce développement exceptionnel du thème traditionnel des travaux imposés magiquement aux démons par Salomon (qui utilise pour cela le pouvoir de son sceau porteur du Nom divin $)^{43}$ fait directement écho aux allusions coraniques à ces démons plongeurs, qui, moins sollicités que les démons constructeurs qui ont participé à l'édification du Temple, sont toutefois censés, selon les commentateurs, rapporter au souverain les perles qui enrichiront le trésor royal ${ }^{44}$.

Ces démons n'ont donc généralement pas pour fonction de satisfaire une curiosité cosmique de Salomon, mais de quérir pour son compte des objets précieux inaccessibles au commun des mortels. Cette motivation n'est pas mentionnée, on l'a vu, dans le conte morisque, où une autre signification est attribuée aux plongées ordonnées par le monarque. Ce développement spécifique du motif coranique est-il pour autant une innovation exclusivement dûe à l'inventivité des conteurs morisques (ou à celle de l'auteur inconnu dont ils ont pu, en l'occurrence, s'inspirer) ? D'autres modèles que les maigres allusions coraniques (et leurs commentaires) semblent avoir joué ici un certain rôle.

43. L. Ginzberg, op. cit., p. 108-111 ; D. Sidersky, op. cit., p. 115-119.

44. Ibid., p. 112, 115. Voir Heidi Toelle, «Des pêcheurs de perles aux Ginn sous-marins », in Dans l'eau, sous l'eau. Le monde aquatique au Moyen Age, D. James-Raoul, C. Thomasset eds., Paris, Pr. de l'Univ. de Paris-Sorbonne, 2002, p. 321-337. 
Le scénario de l'envoi successif de trois démons plongeurs n'est en effet nullement inconnu des folkloristes et des comparatistes. On sait qu'il joue un rôle essentiel dans un mythe Falasha de création de l'homme, ainsi que dans certaines légendes musulmanes (où ce dernier thème est associé à celui de l'attribution à Azrael de la fonction d'Ange de la Mort), et dans plusieurs traditions juives correspondantes, où trois catégories d'anges se distinguent et se hiérarchisent en fonction de l'opinion que leurs représentants ont été amenés à formuler au sujet de ladite création ${ }^{45}$.

Comme l'a bien montré $\mathrm{H}$. Schwarzbaum, ces récits sémitiques constituent solidairement un cycle cohérent, dont le noyau narratif repose sur l'opposition entre les deux premiers des trois génies (ou catégories de génies : ce sont en fait des anges) mandatés, ou interrogés, par Dieu - lesquels échouent dans leur mission (qui consiste à descendre sur terre pour y recueillir la poussière qui servira à créer le premier homme, ou à émettre un avis positif sur cette création) et le troisième, qui est le seul à réussir, ou à savoir donner la réponse attendue par le Créateur.

2) Ces récits apparaissent clairement comme des variations sémitiques sur le thème mythique $d u$ «plongeon cosmogonique », («Earth Diver »), dont on connaît l'ancienneté et la vaste diffusion (Inde, Eurasie, Amérique, Europe Orientale etc. ${ }^{46}$.

L'une des séquences les plus répandues de ce genre de récit, notamment dans les versions européennes, est précisément celle des trois plongeons successifs du démon envoyé par Dieu pour quérir au fond de la mer primordiale le limon qui servira à créer la terre ferme, ou des trois démons plongeurs envoyés successivement au fond des eaux avec la même mission : ce n'est qu'à la troisième

45. H. Schwarzbaum, "Jewish and Moslem sources of a Falasha creation myth", in Studies in Biblical and Jewish Folklore, R. Patai, F. Lee Utley, Dov Noy éds., Bloomington, 1960, p. 41-56.

46. Mircea Eliade, De Zalmoxis à Gengis-Khan. Etudes comparatives sur les religions et le folklore de la Dacie et de l'Europe Orientale, Paris, Payot, 1970, chap. III, «Le Diable et le Bon Dieu : la préhistoire de la cosmogonie populaire roumaine », p. 81-130. Voir François Delpech, «Le plongeon des origines : variations méditerranéennes », Revue de l'Histoire des Religions, CCXVII, 2000 (2), p. 203-256. 
reprise que l'opération sera menée à bien (ou ce n'est que le troisième plongeur qui parviendra à ramener le limon demandé) ${ }^{47}$.

Notre conte morisque n'est certes pas un récit de création de l'homme ni d'instauration de la terre ferme. On voit cependant ce qu'il doit au cycle mythique du plongeon cosmogonique, dont il a manifestement recueilli et transposé le thème le plus caractéristique ${ }^{48}$. D'autres recoupements viennent d'ailleurs conforter la comparaison ici esquissée.

On a vu en effet que, dans le conte, le second démon fait la rencontre d'un ange sous-marin qui lui révèle, historiette à l'appui (le marin dont l'outil est passé par dessus bord cent ans auparavant), que le fond de la mer est inaccessible et que lui-même n'en a pas connaissance. Or il se trouve que cet épisode adventice a justement son équivalent dans certaines versions orientales du mythe de la plongée cosmogonique : on assiste en effet, dans quelques-unes des versions bouriates et votiaks du cycle, à la rencontre de l'oiseau aquatique (envoyé par le dieu suprême en quête du limon sous-marin) et d'une écrevisse qui, à mi-profondeur, lui demande la raison de son voyage et lui explique que, bien qu'ayant vécu depuis plus de cent ans dans la mer, elle n'en a encore jamais vu le fond ${ }^{49}$... Un même substrat mythico-folklorique a donc été de part et d'autre exploité.

On remarquera certes que, dans le conte morisque, ce qui est finalement ramené à la surface, à savoir une coupole de verre avec un jeune homme dedans, n'a rien à voir avec une poignée de limon

47. M. Eliade, op. cit., p. 119 sq. (mythes sud-est asiatiques : l'Esprit Suprême envoie successivement trois animaux en quête du limon sous-marin) et p. 122, n. 134 (thème des « deux essais infructueux suivis d'un troisième, couronné de succès »). Les raisons de l'échec des deux premières tentatives varient selon les versions : erreur ou transgression rituelle de la part du démon plongeur, qui prononce son nom, à la place de celui de Dieu, sur le limon qu'il recueille, ou incapacité d'atteindre le fond.

48. Remarquer qu'il se distingue nettement des versions sémitiques, étudiées par H. Schwarzbaum, par son contexte maritime, et, qu'il n'en a que plus d'affinités avec le cycle de l' «Earth Diver», dont il est, à ma connaissance, le plus évident des parallèles musulmans.

49. Uno Harva, Les représentations religieuses des peuples altaïques, trad. fr., Paris, Gallimard, 1959, p. 76 sq. (voir dans cet ouvrage tout le chapitre «L'origine de la terre », p. 65-79). 
sous-marin, et que cet étrange motif, qui n'apparaît pas dans les récits de plongeon cosmogonique, doit avoir une autre provenance.

C'est probablement le cas, comme on le verra plus loin, mais il appert que la contamination entre le motif en question et le cycle de l' «Earth Diver» n'est pas le bien exclusif du conte morisque : c'est, à défaut d'un bocal de verre, dans une boule d'écume flottant sur les eaux qu'apparaît le démon à Dieu, qui lui ordonnera ensuite de plonger au fond de la mer, dans les légendes de plongeon cosmogonique recueillies en Bucovine et en Pologne ${ }^{50}$.

Tout se passe donc comme si, sur la base d'un mythème assez répandu - celui de la naissance de l'univers, ou du premier homme, à partir d'un Euf Primordial issu de l'Océan originel ${ }^{51}$, s'étaient parallèlement développées diverses légendes, plus ou moins solidaires et interconnectées, dont la portée cosmo (ou anthropo) -gonique initiale se serait plus ou moins bien conservée, ou aurait été occasionnellement oblitérée, ou recyclée et investie d'autres significations $^{52}$.

50. M. Eliade, op. cit., p. 85 et 86. En Estonie le démon sous-marin apparaît à Dieu sous la forme de bulles qui montent à la surface (ibid., p. 87). Dans le monde slave le diable plongeur est parfois enfermé sous la glace, son partenaire (royal ou divin) ayant fait geler la mer après l'y avoir envoyé (ibid., p. 88).

51. Xavier Yvanoff, Mythes sur l'origine de l'Homme, Paris, Errance, 1998, p. 13-35 (« Naissance à partir des œufs »); La Naissance du Monde, Paris, Seuil («Sources Orientales », 1), 1959, passim. Dans plusieurs cas il s'agit plutôt d'une renaissance (après un déluge) que d'une naissance à proprement parler, mais le contexte maritime reste généralement le même. Voir également Venetia Newall, An Egg at Easter. A folklore study, Londres, Routledge and Kegan, 1971, chap. I, «Creation Myths », p. 1-44., et M. Eliade, op. cit., p. 119, où l'auteur mentionne un mythe munda comportant à la fois le motif du plongeon cosmogonique et celui du couple primordial qui, à la suite du déluge, s'est sauvé en se réfugiant dans une gourde qui lui tient lieu d'esquif.

52. Le fait que l'habitacle de verre où est enfermé le jeune homme du conte morisque soit à la fois désigné comme une «coupole»(alcuba) et représenté comme une sorte d'œuf anthropogonique n'est probablement pas dépourvu de portée symbolique : ces deux motifs sont d'évidents symboles cosmiques et représentent respectivement la totalité des orbes célestes et l'omphalos universel (voir note précédente, ainsi que Oleg Grabar, La formation de l'art islamique, trad. fr., $2^{\mathrm{e}}$ éd., Paris, Flammarion, 2000, p. 67-100, en particulier p. 96 sq. et Henry Corbin, Temple et Contemplation. Essais sur l'Islam iranien, Paris, Flammarion, 1980, p. 371, 390 sq., 396). Noter que, lors 
3) En d'autres termes, si les analogies que l'on a relevées entre les mythes de plongeons cosmogoniques et la première partie de notre conte n'impliquent pas nécessairement que ce récit ne soit qu'une variante folklorisée d'un mythe de ce type (dont il n'existe pas, au demeurant, de version spécifiquement musulmane), on peut du moins supposer qu'elles révèlent que le conte a été élaboré (ou réélaboré) dans une région où ces récits cosmogoniques avaient imprégné le folklore courant, par exemple dans les parties islamisées de l'Europe orientale.

Il n'est pas étonnant, du reste, que le personnage de Salomon ait pu servir de support à de semblables contaminations : dans plusieurs légendes rabbiniques le constructeur $d u$ Temple apparaît en effet comme un stabilisateur cosmique, à la fois maître des puissances sous-marines, qu'il scelle d'une pierre gravée au nom de Dieu ${ }^{53}$, possesseur du germe magique issu des abysses grâce auquel les substances les plus résistantes peuvent être percées et taillées ${ }^{54}$, et

de son deuxième voyage, Sindbâd prend à deux reprises l'œuf gigantesque de l'oiseau Rukhkh pour une construction à dôme (Sindbâd de la mer et autres contes. Les Mille et une nuits, contes choisis, t. IV, éd. J. E. Bencheikh et A. Miquel, Paris, Gallimard (Folio), 2001, p. 372 et 422 : ici encore il s'agit probablement de recyclage d'un ancien mythe cosmogonique).

53. J. D. Seymour, op. cit., p. 122 ; Howard Schwartz, Gabriel's Palace. Jewish mystical tales, New York-Oxford, Oxford Univ. Pr., 1993, p. 54 sq. ( «The Waters of the Abyss ») et 283 sq. : l'invasion diluviale des eaux souterraines primordiales, malencontreusement libérées au moment de la fondation du Temple, est finalement stoppée, bloquée par la « Pierre de Fondation » (qui est précisément celle qu'abrite la coupole par excellence qu'est le « Dôme du Roc » : voir Zev Vilnay, Legends of Jerusalem, Philadelphie, Jewish Publ. Soc. of America, 1973, p. 3-36).

54. Voir, parmi les traditions juives relatives au shamir, la légende selon laquelle Satan l'avait soustrait au trône de Dieu, puis caché au fond de la mer où il gisait, incrusté à l'intérieur d'un énorme rocher. Salomon ordonne au Malin de plonger au fond des abysses et de ramener le rocher, lequel, jeté aux pieds du roi, explose, libérant le shamir (J. D. Seymour, op. cit., p. 117 sq.) : voir Adela Goodrich-Freer, "The powers of Evil in Jerusalem", Folklore, XVIII, 1907, p. 54-76 (p. 74 sq.), et Moses Gaster, "The legend of Merlin", Folklore, XVI, 1905, p. 407-426 (p. 419 sq.), qui cite une variante roumaine assez déformée où le rôle de Satan est tenu par l'Archange Gabriel, mais dont le scénario semble se situer à mi-chemin entre la légende d'Asmodée et celle de Merlin (où l'épisode des dragons souterrains qui empêchent la construction de la Tour de Vortigern reprend le motif cosmogonique évoqué dans la note précédente). 
garant de l'équilibre axial d'un univers constamment menacé de crouler sur ses propres fondations ${ }^{55}$. La construction du Temple fait donc de Salomon une sorte de héros cosmogonique.

Toute coupole n'est par ailleurs qu'une répétition de la Mère des coupoles qu'est le « Dôme du Rocher », lequel n'est à son tour que l'accomplissement du Temple, dont il couronne la «Pierre de Fondation ${ }^{56}$. Celle-ci est enfin le sceau de la Mer intérieure, c'està-dire du royaume chaotique de Léviathan, sous-jacent au Temple, centre de l'univers ${ }^{57}$.

On voit comment d'un complexe mythique aussi cohérent, aussi ancré dans un paysage originaire, ont pu par diffraction procéder des configurations imaginaires qui, dans d'autres contextes, ont abouti à des récits aussi atypiques et apparemment aberrants que notre conte morisque. On y retrouve bien le chaos maritime, le roi constructeur maître des démons, la mystique coupole verte, le plongeon paracosmogonique, mais tout ce matériel mythique a été désarticulé, remonté en forme de conte sapiential, réinvesti dans une histoire qui ne relève plus de la Geste primordiale mais du temps de la tribulation et de son corollaire, le hors-temps paradisiaque.

\section{Pêches miraculeuses et talismans de Souveraineté}

1) Le second motif digne de retenir notre attention est celui de la mystérieuse alcuba de verre verte, « coupole» devenue habitacle monoplace ou sous-marin de poche. Cet introuvable objet transporté sur la grève par les démons-fonctionnaires rappelle bien d'autres pêches miraculeuses. Ce n'est pas la première fois en effet que

55. Selon le Testament de Salomon, le constructeur du Temple oblige deux démons à maintenir suspendu dans les airs un pilier qui ne chutera qu'à la fin du monde (J. D. Seymour, op. cit., p. 132 sq.). Ce mythe est probablement à l'origine de la légende médiévale selon laquelle la «Pierre de Fondation » abritée par le Dôme du Roc s'élève occasionnellement dans les airs (ou se mettra à léviter à l'arrivée du Messie) : Z. Vilnay, op. cit., p. 23 sq.

56. O. Grabar, op. cit., p. 76 sq. ; H. Corbin, op. cit., loc. cit. ; Z. Vilnay, op. cit., p. 17-36.

57. Ibid., p. 8 sq. et p. 19 ; H. Schwartz, op. cit., loc. cit. 
Salomon voit surgir des flots un article précieux dont il saura éventuellement tirer parti. On a vu que le shamir lui vient ainsi des profondeurs ${ }^{58}$. On sait que le Sceau divin, où réside le pouvoir que le roi magicien exerce sur les démons, a aussi quelque affinité maritime : selon certaines versions de la légende, il a été trouvé dans le ventre d'un poisson par l'Ange Gabriel ; selon d'autres récits, il a été récupéré de même, après que le roi des démons l'eut volé à Salomon et jeté à la mer ${ }^{59}$. Dans plusieurs contes arabes il est d'ailleurs question de pêcheurs ou de plongeurs qui ramènent au roi l'anneau (souvent magique, ou du moins lié, d'une manière ou d'une autre, à la Souveraineté) qu'il a perdu en mer ${ }^{60}$. On voit mal, a priori, comment l'habitacle de verre du conte morisque pourrait procéder de ces légendes, dont il ne se rapproche que par sa provenance marine.

D'autres récits évoquent cependant, parmi les objets magiques associés à Salomon, la coupe panoptique qui lui permettait de surveiller à distance les activités des êtres vivants et des démons ${ }^{61}$. Il s'agit bien cette fois d'un objet de verre, dont la forme aurait pu suggérer l'image de cette mystérieuse alcuba de verre verte évoquée par le conte. Cette coupe magique, manifestement héritée

58. Voir supra n. 54.

59. R. Basset, « Salomon dans les légendes musulmanes, chap. VI, «Les objets merveilleux de Salomon», par. 7, «L'anneau et le sceau », Revue des Traditions Populaires, VII, 1892, p. 377 sq. Cf. Karl Preisendanz, « Salomo », in Pauly-Wissowa, RE, Suppl. VIII, 1956, col. 670. Pour la légende de la récupération de l'anneau volé, telle que l'ont transmise les textes morisques, voir A. Vespertino Rodríguez, op. cit., p. 264 et Ph. Roisse, op. cit., p. 396 (pendant sa période de "pénitence » forcée Salomon est entré au service de modestes pêcheurs). Selon D. Sidersky, op. cit., p. 121, cette histoire d'anneau avalé par un poisson, absente des sources juives, serait une innovation imputable aux remanieurs arabes de la légende.

60. Voir Claude Brémond, « Mille contes en un » $\left(=2^{\mathrm{e}}\right.$ partie du «Dossier d'un conte des Mille et une nuits »), Critique, CCCXCIV, 1980, p. 254-272 (à propos du conte d'Abu Qir et d'Abu Çir). Voir aussi D'Herbelot, Bibliothèque Orientale, III, 131 sq. (l'anneau d'Harun al Rashid). Il n'y a pas lieu d'examiner ici les rapports de ces histoires avec le thème de «l'anneau de Polycrate » ou avec le récit du plongeon de Thésée : voir F. Delpech, op. cit., p. 231-237.

61. R. Basset, op. cit., par. 6, « La coupe », Revue des Traditions populaires VII, 1892, p. 165. 
de la légende de Djemshid ${ }^{62}$, a-t-elle été confondue avec un autre attribut du mythique roi iranien, ce char de cristal volant qui lui permettait de visiter les orbes célestes (plus prosaïquement, les légendes salomoniennes l'ont transformé en tapis volant) ${ }^{63}$ ? Il faut néanmoins reconnaître qu'aucun de ces deux objets n'a de provenance ou affinité maritime.

Remarquons toutefois que la coupe magique et la voiture de verre, qui sont complémentaires en tant qu'instruments d'investigation et de domination cosmocratique, relèvent communément et indirectement de la catégorie des «talismans de souveraineté ».

Or on sait que dans le monde iranien, notamment dans le complexe mythique avestique où s'enracine la légende de Yima-Djemshid, le principe de la souveraineté, avant de se matérialiser dans des objets symboliques, est représenté par l'intangible « Gloire Lumineuse », principe igné et métaphysique, que les candidats à la royauté ne peuvent posséder que s'ils sont mystiquement « qualifiés » ${ }^{64}$.

Quoique liée au feu, cette « Gloire Lumineuse » réside au fond des eaux, au sein de la mer Vourukasa, où l'a cachée Apam Napât, le « descendant des eaux », alors qu'elle était l'enjeu d'un combat entre le Saint Esprit et l'Esprit Mauvais ${ }^{65}$. Le candidat à la souveraineté doit donc, pour s'en emparer, plonger dans les profondeurs de cette mer : il ne parviendra toutefois à ses fins que s'il a la qualification

62. Arthur Christensen, Les types du Premier Homme et du Premier Roi dans l'histoire légendaire des Iraniens, $2^{\mathrm{e}}$ partie «Jim», Leyde, Archives d'Etudes Orientales, 14 (2), 1934, p. 128-137 (« La coupe magique »). Sur le transfert de cette coupe à la légende de Salomon voir ibid., p. 119, 132, et R. Basset, loc. cit.

63. R. Basset, op. cit., par. 4, « Le tapis », RTP, VI 1891, p. 610-612. Sur le char de verre de Djemshid, qui n'est autre que le Yima de l'ancienne tradition avestique, voir Georges Dumézil, Mythe et Epopée II. Types épiques indoeuropéens : un héros, un sorcier, un roi, Paris, Gallimard, 1971, p. 310-312 ( «Yima, le char de cristal et la fête du Nouvel An ») et, pour l'équivalent indien de Yima, p. 306 sq. ( « Vasu Uparicara, le char de cristal et la fête de la Guirlande d'Indra »). Voir également A. Christensen, op. cit., p. 86.

64. G. Dumézil, op. cit., p. 219 sq., 282-289, 326 sq.

65. G. Dumézil, Mythe et Epopée III - Histoires romaines, Paris, Gallimard, 1978, p. 24-27. Voir également Gherardo Gnoli, « Un particolare aspetto del simbolismo della luce nel Mazdeismo e nel Manicheismo », Ist. Univ. Orientale di Napoli, Annali, n. s., XII, 1962, p. 95-128 (p. 98-105). 
requise (notamment la condition de bon Arya), car cette gloire, dans le cas contraire, s'esquivera, entraînant l'eau derrière elle, et la mer s'agrandira pour lui permettre d'échapper au nageur. Telle est la mésaventure qui survint au touranien Franrasyan, qui, à trois reprises, s'immergea dans cette mer, y poursuivit en vain la «Gloire» toujours fuyante, sans jamais réussir à l'atteindre ${ }^{66}$.

Ce complexe d'images et de légendes, dont G. Dumézil a montré qu'il relève d'une mythologie des eaux indo-européennes, fondée sur le thème du «feu dans l'eau » ${ }^{67}$, est sans doute à mettre en parallèle avec tous les récits iraniens (et post-iraniens) d' « ordalies de souveraineté » où l'épreuve décisivement qualifiante consiste dans le franchissement ou le «domptage » d'une étendue d'eau ${ }^{68}$.

2) Bien que les significations respectives soient toutes différentes, on voit en quoi ce mythe du «plongeon pour la Souveraineté » se rapproche, au niveau des schèmes narratifs et imaginaires mobilisés, des traditions sur le «plongeon cosmogonique » évoquées plus haut : on remarque notamment, de part et d'autre, la récurrence du motif des «trois tentatives », qui est justement celui que l'on retrouve dans le conte morisque ${ }^{69}$. Il y a probablement eu des interférences

66. G. Dumézil, ME III, p. 26, 31-33.

67. La bibliographie du thème s'est beaucoup enrichie ces dernières années. Pour une synthèse récente voir D. Briquel, « Le thème indo-européen du feu dans l'eau. Application en Grèce », in L'Eau et le Feu dans les religions antiques, G. Capdeville éd., Paris, De Boccard, 2004, p. 11-23.

68. Luc Desnier, De Cyrus le Grand à Julien l'Apostat. «Le Passage du Fleuve ». Essai sur la légitimité du Souverain, Paris, L'Harmattan, 1995.

69. Noter que le schéma ternaire du mythe cosmogonique implique, comme on l'a vu, deux échecs suivis d'une réussite finale; la légende de Franrasyan suppose, quant à elle, trois échecs successifs et définitifs. Le conte morisque opère en quelque sorte la difficile synthèse entre ces deux formules : il y a bien échec des deux premières tentatives puisque aucun des deux démons dépêchés par Salomon ne parvient à atteindre le fond de la mer. À la troisième reprise (à vrai dire mal individualisée puisqu'elle est imputée au même deuxième démon, qui a été obligé, dans un premier temps, de constater son impuissance à accomplir la mission demandée, vu que le conteur a " oublié » qu'un troisième démon avait été initialement prévu...), le résultat est à la fois un échec, puisque le fond reste inaccessible, et une (demi) réussite, puisqu'il finit bien par trouver quelque chose d'intéressant... L'omission du troisième démon tient peut-être justement à une difficulté des conteurs à unifier une tradition qui était éventuellement représentée par des versions initiales multiples et contradictoires... 
entre ces deux cycles hétérogènes, l'un essentiellement iranien, l'autre surtout « eurasiatique », notamment dans les cultures tributaires de ce double héritage : on voit par exemple, dans l'ésotérisme kurde, s'associer à un contexte cosmogonique le motif du plongeon comme quête d'un objet lumineux (en l'occurrence une perle mystique liée à la Souveraineté divine et à l'origine du premier Archange $)^{70}$.

Les «talismans de souveraineté » sont tout particulièrement des avatars matérialisés de cette «Gloire Lumineuse », dont ils sont à la fois les symboles et les supports, ce qui explique qu'ils soient, comme elle, si souvent censés provenir des abîmes marins (d'où la nécessité de traverser les mers ou d'y plonger pour les conquérir) ${ }^{71}$ et rend compte de leur fréquente association à la lumière ${ }^{72}$ ou à des matières translucides, comme le verre ${ }^{73}$.

70. Mohammad Mokri, «Le symbole de la perle dans le folklore persan et chez les Kurdes Fidèles de Vérité (Ahl - E Haqq) », Journal Asiatique, CCXLVIII, 1960, p. 463-481. Voir aussi Nûr Alî-Shâh Elâhî, L'Ésotérisme kurde. Aperçus sur le secret gnostique des Fidèles de Vérité, M. Mokri éd., Paris, Albin Michel, 1966, p. 13-24.

71. Bruce Louden, "Bacchylides 17: Theseus and indo-iranian Apâm Napât", Journal of Indo-European Studies, XXVII, 1999, p. 57-78. Voir Atsuhiko Yoshida, «Sur quelques coupes de la fable grecque », Revue des Études Anciennes, LXVII, 1965, p. 31-41.

72. La légende de l'origine maritime du Sceau de Salomon (et du shamir) est à rapprocher du motif de l'escarboucle lumineuse avec laquelle, pour le compte d'Alexandre, Khadir éclaire le chemin à travers le Pays des Ténèbres, en quête de la Fontaine de Vie: selon certaines rédactions du Roman d'Alexandre, cette pierre a été trouvée dans l'estomac d'un poisson (voir A.R. Nykl, op. cit., p. 562 et Jacques Lacarrière, trad., La légende d'Alexandre, Paris, Gallimard (Folio), p. 141). Remarquer que, dans les anciennes versions russes de la légende d'Alexandre, on retrouve le même motif (voir Alfred Nicolas Rambaud, La Russie Épique. Étude sur les chansons hérö̈ques de la Russie, Paris, Maisonneuve, 1876, p. 405 ; cf. p. 143) et que ce dernier est passé dans la légende russe de Salomon (ibid., p. 387 : Salomon qui s'est embarqué, d'abord comme cuisinier puis comme marchand, rapporte de ses voyages maritimes une pierre qui brille toute seule). Cette pierre lumineuse, qui rappelle celle qui aurait éclairé Jonas à l'intérieur de la baleine, reconduit manifestement au même type de symbolisme que la «perle » cosmogonique des «Fidèles de Vérité » kurdes : M. Mokri («Le symbole de la perle...», p. 475) mentionne une légende, tirée du Livre des Secrets de Hamza, manifestement inspirée d'un épisode célèbre du Roman d'Alexandre (sur lequel nous reviendrons plus loin), où un plongeur descendu sous les eaux dans une sorte de bathyscaphe est avalé avec son engin par un requin, dans le ventre duquel il 
La mystérieuse alcuba que les démons extirpent des flots pour Salomon pourrait donc bien provenir de cette zone d'interférence entre la mythologie iranienne de Yima-Djemshid (dominée par le thème de la Gloire Lumineuse) et le répertoire légendaire plus traditionnellement attaché à la figure du roi hébreu, maître des éléments et des génies, constructeur du Temple et garant de la stabilité de l'univers. La curiosité maritime du souverain, les plongeurs qu'il commissionne, font partie d'un plan de conquête, d'extension ou de réaffirmation de la Souveraineté cosmocratique. L'étrange objet qui lui est rapporté - dont il y a lieu de penser que, transparent ou opaque, il est associé, par ses deux «vetas » (blanche et noire), à l'obscure clarté d'une insaisissable lumière sous-marine ${ }^{74}$ - pourrait prendre rang, comme le sceau, le shamir, la coupe magique, parmi les objets talismaniques, susceptibles de donner corps à la "Gloire Lumineuse », et serait indispensable donc à la panoplie du souverain.

On sait, notamment grâce aux travaux d'Henry Corbin, qu'une partie de cette mythologie païenne préislamique a été « récupérée »

découvre une perte lumineuse. On voit dans cet exemple un cas intéressant de contamination entre la légende alexandrine et la symbolique ésotérique d'une secte iranienne (où mystique et cosmogonie s'interpénètrent). Ce genre de contamination semble supposer une référence commune à l'ancien thème avestique de la résidence aquatique de la «Gloire Lumineuse ».

73. On a vu plus haut ce qu'il en est des coupes magiques de Djemshid et de Salomon (n. 62) et du char de cristal de Yima et Vasu Uparicara (n. 63). Nous avons également noté le rôle que le verre joue dans certaines légendes salomoniennes (le couvercle de verre utilisé pour s'emparer du shamir: n. $25 ; c f$. le parquet de verre semblable à de l'eau que Salomon fait traverser à la reine de Saba pour l'obliger à soulever sa robe et montrer ses jambes dans les versions arabes; le motif se retrouve dans certaines versions du Roman d'Alexandre, où c'est le palais de la reine Candace qui est ainsi équipé : voir Corinne Jouanno, Naissance et métamorphoses du Roman d'Alexandre, Paris, CNRS, 2002, p. 320-323).

74. Un trait analogue figure dans les légendes des Parsis relatives aux sept «talismans » créés par Djemshid et détruits par Alexandre : l'une de ces merveilles est une coupole à moitié blanche, à moitié noire : les âmes des morts y apparaissent trois jours après leur décès, se montrant sur la moitié blanche s'ils sont voués au paradis, sur la moitié noire si l'enfer les attend (A. Christensen, op. cit., p. 77) : cf. supra n. 15 et infra n. 101. Comme le Salomon de notre conte morisque, Djemshid envoie des divs plongeurs chercher pour lui les richesses du monde sous-marin : ibid., p. 68, 90, 97. 
et spiritualisée par l'Iran shiite et que c'est ce patrimoine mythique qui a servi à alimenter la légende d' $\mathrm{Al} \mathrm{Khadir}^{75}$ : cet éternel errant, spécifiquement lié aux espaces maritimes, apparait, on l'a vu, comme le manipulateur privilégié du talisman lumineux (d'origine aquatique) qui guide Alexandre à travers les ténèbres ${ }^{76}$, et certaines légendes font même de lui un plongeur qui cherche à atteindre le fond de la mer $^{77}$. Cette double affinité, aquatique et ignée, indique que le folklore relatif à ce personnage suppose un recyclage partiel de l'ancienne mythologie de la « Gloire Lumineuse », laquelle semble également avoir laissé des traces dans certaines traditions gnostiques, dans plusieurs sectes para-shiites iraniennes ${ }^{78}$ et peut-être dans quelques textes apocryphes de l'Europe Orientale, là où précisément ont fleuri les légendes de "plongeons cosmogoniques ${ }^{79}$.

Notre récit morisque ne comporte certes aucune allusion explicite à cet arrière-plan néo-iranien : il n'est en apparence concerné ni par des représentations cosmogoniques ni par une problématique de la souveraineté. Le Salomon qu'il met en scène est néanmoins l'indirect mais inconstestable héritier d'une mythologie : les connotations symboliques de l'étrange objet que des profondeurs maritimes lui

75. H. Corbin, Terre Céleste et Corps de Résurrection. De l'Iran mazdéen à l'Iran shî'ite, Paris, Buchet-Chastel, 1960, p. 240-250. Voir également, du même auteur, En Islam iranien. Aspects spirituels et philosophiques, Paris, Gallimard, t. II, 1971, chap. III, « La Lumière de Gloire mazdéenne (Xvarnah) et l'angélologie », p. 81-140, et chap. IV, « La Lumière de Gloire et le Saint Graal », p. 141-210, où sont examinés les prolongements du thème de la Gloire Lumineuse dans l'Islam shiite iranien.

76. Voir supra n. 72.

77. Angelo Piemontese, «Le submersible Alexandrin dans l'abysse, selon Amir Khusrau », in Alexandre le Grand dans les littératures occidentales et proche-orientales, L. Harf Lancner, C. Kappler, F. Suard éds., Univ. Paris XNanterre : Littérales (h.s.), 1999, p. 253-271 : voir p. 256, n. 8. Voir supra n. 39 et infra n. 100.

78. Voir supra n. 70. Comparer avec l'Hymne de la Perle des Actes apocryphes de Thomas : voir Nadia Ibrahim Frederikson, « La perle, entre l'océan et le ciel. Origines et évolution d'un symbole chrétien », Revue de l'Histoire des Religions, CCXX, 2003 (3), p. 283-317.

79. Voir les rôles respectifs joués par l'archange Gabriel dans la cosmogonie ésotérique kurde des "Fidèles de Vérité » (N.A. Elâhî, op. cit., p. 19-26) et dans la légende apocryphe roumaine citée par M. Gaster, op. cit. (voir supra n. 54). 
rapportent les démons plongeurs révèlent que cette mythologie continue à sous-tendre de manière latente tout un imaginaire que la pensée sapientiale reconduit sans en effacer totalement les implications mystiques $^{80}$.

\section{UN JEUNE HOMME ENCAPSULÉ}

1) C'est toutefois plus qu'un objet talismanique que les démons viennent jeter aux pieds de Salomon : ce qui en sort vient aussitôt capter l'attention et déplacer la portée du geste. S'il n'y a rien d'extraordinaire dans le fait de trouver une bouteille dans la mer, il est certes moins banal d'en voir sortir quelqu'un.

L'aventure n'est cependant pas sans exemple, dont le plus notoire est bien sûr l'extirpation, occasionnelle ou délibérée, des génies rebelles enfermés par Salomon dans des jarres scellées de plomb gravé au Nom de Dieu et enterrées ou jetées au fond des eaux.

On sait que le thème est ancien, que le mentionnent le Testament de Salomon, les textes gnostiques de Nag Hammadi et toute une série de textes arabes dont le plus connu est le conte du pêcheur et du génie des Mille et une Nuits ${ }^{81}$. La communauté morisque en a eu connaissance, comme en témoignent les versions aljamiadas du conte de la Ville de Cuivre, dont on sait qu'il se termine, dans sa forme canonique (qui figure également dans les Mille et une Nuits), par la séquence de la libération, en présence du khalife, des génies enfermés dans les jarres ramenées d'Occident par l'émir Musa, lequel les

80. Il y aurait lieu de développer la comparaison entre le matériel imaginaire mobilisé par le conte morisque et la mythologie avestique de la «Gloire Lumineuse » : la nourriture blanche apportée au mancebo par l'oiseau merveilleux rappelle par exemple le haoma blanc produit par l'Arbre de Vie qui pousse dans la mer Vourukasa, et le rôle déterminant joué par la mère du héros semble rappeler celui de la déesse des eaux Anâhitâ, détentrice originelle et privilégiée d'une partie de la «Gloire Lumineuse » (voir G. Gnoli, op. cit., loc. cit.).

81. Voir Israel Levi, "Le conte du "Diable dupê" dans le folklore juif », Revue des Etudes Juives, LXXXV, 1928, p. 137-163, et F. Delpech, « En torno al Diablo Cojuelo : demonología y folklore », in El Diablo en la Edad Moderna, M. Tausiet, J. S. Amelang éds., Madrid, Marcial Pons, 2004, p. 99-131. 
avait fait quérir par des pêcheurs noirs dans la mer où elles avaient été jetées.

Il faut bien reconnaître que, si les concepteurs du conte morisque se sont souvenus de ce cycle de légendes (ce qui est d'autant plus vraisemblable que Salomon en est, dans les deux cas, un protagoniste essentiel), ils ont fait plus que le recycler : ils en ont complètement subverti le sens. Salomon joue dans le texte aljamiado un rôle contraire à celui qui est le sien dans la légende habituelle, et le jeune homme sorti de la cuve de verre n'a rien d'un démon : il n'a pas été placé là en punition d'une quelconque rebellion, mais pour vivre dans un état semi-angélique, loin des tentations du monde. Il n'a d'ailleurs, contrairement aux démons scellés par le roi-magicien, aucune envie de sortir de son flacon, qu'il s'empresse de réintégrer, une fois terminé son dialogue avec le roi hébreu. Tout se passe donc comme si son histoire avait été délibérément conçue comme une sorte d'antithèse de celle des démons embouteillés.

Elle en évoque en effet, mais a contrario, les connotations eschatologiques : si la libération des démons scellés dans les jarres préfigure dans une certaine mesure le déchaînement apocalyptique de Gog et Magog, l'image du jeune homme isolé dans son bocal fait écho au sommeil mystique des Sept Dormants, figure de l'attente bienheureuse de la Parousie.

Un autre indice de la solidarité antithétique des deux récits nous est encore une fois indirectement fourni par une occurrence du mystérieux Al Khadir : le conte de la Ville d'Airain spécifie en effet que la mer du bout du monde, où les plongeurs noirs vont pêcher, pour Musa, les jarres où sont reclus les génies rebelles, est régulièrement visitée par le Prophète Verdoyant, lequel, glissant sur les eaux, a converti à la Vraie Foi ses païens riverains ${ }^{82}$.

Epiphanie cyclique qui ne va pas sans rappeler les quotidiennes apparitions de l'oiseau-ange qui, dans le conte morisque, apporte

82. Voir la thèse inédite de Michael Barry, Problèmes d'approche des Mille et une nuits et la problématique du conte de la Cité d'Airain, Paris, EHESS, 2004, et F. Corriente Córdoba, op. cit., loc. cit. 
au jeune renonçant la mystique pitance qui lui permet de vivre en complète et sublime autarcie ${ }^{83}$.

2) Un homme à la mer, enfermé dans un habitacle de verre : au-delà des génies scellés dans les jarres, un autre mythe, un autre modèle, s'impose également à l'esprit, trop célèbre pour que le conteur ait pu ne pas y penser. Il s'agit bien sûr de l'exploration sous-marine tentée par Alexandre dans sa cloche de verre, qui, depuis le pseudoCallisthène, égaie les lecteurs de presque toutes les versions du fameux Roman ${ }^{84}$.

On sait que, dans le texte grec, c'est pour s'emparer des perles qui gisent au fond de la mer qu'Alexandre imagine son bathyscaphe : la tentative échoue, l'engin, secoué par les poissons, ayant dû, à deux reprises, être ramené à la surface, pour être finalement gobé puis régurgité par un monstre marin, qui crachera sur la grève l'explorateur intact et épouvanté, bien décidé à ne pas renouveler l'exploit ${ }^{85}$.

Les multiples versions du Roman insistent, à propos de cet épisode, tantôt sur la cupidité ou le fol orgueil d'un héros transgresseur qui veut sonder les mystères inaccessibles à l'homme, tantôt, bien au contraire, sur la grandeur intellectuelle et militante d'un Cosmocrator qui, devenu par anticipation héraut de l'Islam, découvre émerveillé l'immensité et la magnificence d'un univers qu'il a pour mission

83. Comparer avec les quotidiennes visites de l'oiseau Sîmurgh à la coupole du Paradis dans l'un des contes recueillis par Auguste Bricteux, Contes Persans (ms. inédit de Berlin), Paris-Liège, 1910, n X, p. 305-344 (voir p. 329-331). Cf. supra n. 52 et infra n. 104.

84. Sur cet épisode voir David John Athole Ross, Studies in the Alexander Romance, Londres, Pindar Pr., 1985, chap. XXIII, "Alexander and the faithless lady: a submarine adventure", p. 382-403 ; Catherine Gaullier-Bougassas, "La réécriture inventive d'une même séquence : quelques versions du voyage d'Alexandre sous la mer », Bien dire et bien aprandre, XIV, 1996, p. 7-19; A. Piemontese, op. cit. ; C. Jouanno, op. cit., p. 272 ; Sandrine Hériché, « Immersion et survivance dans Les faicts et les conquestes d'Alexandre le Grand de Jehan Wauquelin ( $\mathrm{XV}^{\mathrm{e}}$ siècle) », in Dans l'eau, sous l'eau. Le monde aquatique au Moyen Age (cf. supra, n. 44), p. 339-355.

85. Pseudo Callisthène, Le Roman d'Alexandre, trad. G. Bounoure, B. Serret, Paris, Les Belles Lettres, 1992, II, 38, p. 80-82. 
d'assujettir à la Vraie Foi ${ }^{86}$. Ce thème de l'explorateur sous-marin dans sa cage de verre, qu'il s'agisse d'Alexandre ou d'un autre personnage, a eu, sous l'influence du Roman d'Alexandre, une large diffusion, notamment dans le folklore juif ${ }^{87}$ et en Europe centrale et orientale ${ }^{88}$.

Il faut ici remarquer que c'est précisément à Salomon que l'aventure a été attribuée en Bulgarie ${ }^{89}$, en Russie ${ }^{90}$ et en Ukraine ${ }^{91}$, probablement à partir de traditions apocryphes byzantines : dans ces récits le résultat de l'exploration est tantôt négatif (comme dans le modèle grec), Salomon ne parvenant pas à atteindre le fond de la mer et se voyant contraint à remonter à la surface ${ }^{92}$, tantôt positif, lorsqu'il profite de l'occasion pour s'approprier des talismans ${ }^{93}$ ou pour tirer de l'observation des habitudes des poissons une leçon de stratégie et de politique ${ }^{94}$.

86. Sur l'image musulmane d'Alexandre voir François de Polignac, «L'image d'Alexandre dans la littérature arabe : l'Orient face à l'Héllenisme », Arabica, XXIX, 1982, p. 296-306; id., «L'homme aux deux cornes. Une image d'Alexandre du symbolisme grec à l'apocalyptique musulmane », Mélanges de l'Ecole Française de Rome-Antiquité, XCVI, 1984 (1), p. 29-51. Pour une version hispano-arabe voir Emilio García Gomez éd., Un texto árabe occidental de la leyenda de Alejandro, Madrid, Inst. de Valencia de Don Juan, 1929, (p. 56-61 pour l'épisode sous-marin). Sur les diverse interprétations de l'exploration subaquatique voir les études citées supra n. 84 .

87. Voir Stith Thompson, Motif-Index of Folk-Literature, CopenhagueBloomington-Indianapolis, Indiana Univ. Pr.-Rosenkilde and Bagger, 19551958, motif F 1022-1, Descent into ocean in glass box.

88. Ibid., motif L 414.1, "King vainly attempts to measure the height of the sky and the depth of the sea » (version lithuanienne).

89. J.D. Seymour, op. cit., p. 93 sq. (d'après les Légendes religieuses bulgares, de Lydia Schischmanoff, 1896, p. 84).

90. A. Rambaud, op. cit., p. 399.

91. J.D. Seymour, op. cit., p. 53 (d'après Eugène Hins, «Les légendes chrétiennes de l'Ukraine », Revue des Traditions Populaires, II, 1887, p. 513 et 517).

92. Ibid., p. 513. Voir Seymour, op. cit., p. 53 et 93 sq.

93. A. Rambaud, op. cit., p. 399 (Salomon, qui ne peut remonter à la surface par suite de la trahison de sa femme, a été repêché par des démons qui se disputent la possession d'un talisman qu'ils ont dérobé et lui demandent de les départager : Salomon parvient à les tromper et à s'approprier le talisman).

94. J.D. Seymour, op. cit., p. 93 : Salomon apprend à organiser ses armées en imitant les pratiques des poissons. Ce motif vient tout droit des versions médiévales du Roman d'Alexandre (voir C. Gaullier-Bougassas, op. cit.). 
Le rapport de notre conte morisque avec cette tradition est évident. L'envoi de démons explorateurs y a remplacé, par délégation, la descente personnelle du monarque dans les profondeurs sous-marines, mais c'est bien de la tradition alexandrine que vient le motif de la curiosité cosmique, du désir de sonder les mers.

De même, c'est encore aux versions médiévales - musulmanes et chrétiennes - du Roman d'Alexandre qu'a été manifestement empruntée l'observation du premier démon plongeur au sujet de la « loi des poissons » et de la prépotence des plus forts : le récit vise, selon les cas, à dénoncer cette tyrannie, ou à en tirer, au bénéfice du souverain, un modèle utile de stratégie de domination, voir un enseignement relatif aux moyens d'équilibrer la force par la ruse ${ }^{95}$.

Il est enfin révélateur que l'épisode de la rencontre du deuxième démon avec un ange (qui lui raconte l'histoire du marin qui a perdu son outil et lui fait comprendre l'inutilité de son exploration) ait son équivalent exact dans la légende ukrainienne : Salomon n'y parvient pas, dans sa cloche de verre, à atteindre le fond; il rencontre un homard géant qui lui dit que l'océan est insondable et lui avoue qu'il a lui-même pendant vingt ans essayé en vain d'en mesurer la profondeur, lui conseillant finalement de remonter à la surface, de peur qu'un de ses congénères n'entreprenne de couper avec ses pinces la chaîne qui le relie au bateau ${ }^{96}$. Nous avons vu qu'un épisode tout à fait analogue figure dans le cycle eurasiatique du «plongeon cosmogonique ${ }^{97}$, ce qui confirme la solidarité, la complexité et l'ampleur du réseau «folklorique » auquel se rattache le conte morisque, dont on aurait pu croire, à première vue, qu'il n'était qu'un hapax dû à la gratuite fantaisie d'un conteur particulièrement imaginatif...

95. Voir note ci-dessus. Sur ce point la version bulgare citée par J. D. Seymour (p. $93 s q$.) est identique aux versions médiévales françaises et espagnole du Roman.

96. E. Hins, op. cit., p. 513 (= J. D. Seymour, p. 53).

97. Voir supra n. 49 (à propos de l'écrevisse des versions bouriates et votyaks). 
3) Si la référence à la légende alexandrine (et à ses variantes «salomonisées ») est donc évidente, il n'en reste pas moins que, comme on l'a vu dans le cas de la comparaison avec le cycle des « démons embouteillés », le conteur morisque déconstruit ou croise ses modèles et en déplace les composantes.

Pour mieux mettre en relief la portée sapientiale de l'expérience, le conteur en démultiplie les protagonistes : ce n'est plus le souverain qui effectue lui-même l'exploration sous-marine, déléguée à des génies plongeurs, et le motif du bathyscaphe de verre est reporté sur un nouveau personnage, le jeune homme encapsulé, lequel ne cherche pas à sonder les abîmes, mais s'y réfugie pour fuir le monde, ce qui confère au bocal vitreux, qui n'est plus un instrument de prospection, mais de protection, une fonction, toute nouvelle, d'antichambre paradisiaque.

Or cette bifurcation nous fait, encore une fois, recouper l'un des cheminements d'Al Khadir. Le nouveau personnage qu'introduit le conte morisque, dont on a vu qu'il est assisté par les anges et quotidiennement visité par un oiseau merveilleux qui lui apporte une nourriture divine, n'évoque pas seulement le héros macédonien parce qu'il partage avec lui le motif de l'habitacle de verre sousmarin. Dans plusieurs versions musulmanes du Roman, Alexandre est en effet accompagné dans sa cage de verre par l'" Ange de la $M e r »$, qui guide et protège son exploration, le nourrit d'une grappe merveilleuse et lui permet de revenir sain et sauf à bon port avec une magique vélocité ${ }^{98}$.

Cet ange marin semble bien être un proche, voire une sorte d'alter ego de Khadir, dont on a déjà rappelé les rapports avec Alexandre ${ }^{99}$, et dont on sait que, selon Mohammad Hamadâni (XII siècle), il avait lui aussi vainement tenté d'atteindre le fond de la mer de Chine

98. A. Piemontese, op. cit., p. 261-263. Voir E. García Gomez, op. cit., loc. cit., où l'on retrouve les mêmes détails (grappe merveilleuse et retour magique au point de départ). Cet Ange de la Mer figure aussi dans la version éthiopienne (Ernest Alfred Tompson Wallis Budge, The Alexander Book in Ethiopia, Cambridge, 1933, p. 169-172).

99. Voir supra nn. 39 et 72. 
et avait été à cette occasion admonesté par un ange qui lui avait raconté l'échec d'une tentative identique anonymement entreprise à l'époque de Noé ${ }^{100}$. Cet épisode a une évidente analogie avec le dialogue du deuxième démon plongeur et de l'ange dans le conte morisque, ce qui nous permet de boucler la boucle et de réaffirmer la cohérence du système de représentation qui soustend tout cet ensemble de récits distincts mais traversés de motifs récurrents.

\section{UNE ABSCONDITIO PRÉ-PARADISIAQUE}

1) Pour reconstituer tout le réseau « folklorique » auquel reconduisent, chacun à son niveau et selon sa ligne spécifique, ces multiples récits plus ou moins clairement apparentés, il faudrait - ce qu'il n'y a pas lieu de faire ici - suivre en détail d'autres pistes comparatives.

Retracer par exemple, en amont, sur fond de représentations iraniennes (recyclées par le judaïsme alexandrin, les sectes gnostiques puis shiites de la Perse et finalement par l'ensemble de l'ésotérisme musulman), ce qui rapproche notre récit ibérique du conte de Buluqiya - où la quête trans-maritime du héros le met en présence d'anges marins, d'un oiseau pourvoyeur de nourritures merveilleuses et finalement de Khadir en personne ${ }^{101}$-, des légendes judéo-alexandrines relatives aux communautés " cachées » de sages (les Gymnosophistes, les Réchabites) vivant aux confins du monde dans un état semiparadisiaque analogue à celui des habitants du vara de l'avestique

100. A. Piemontese, op. cit., p. 256, n. 8 (voir supra n. 77). Dans la version d'Amir Khusrau, Al Khadir (Xezr) fait partie de l'expédition sous-marine d'Alexandre : ibid., p. 266.

101. Voir L. López Baralt, op. cit., et Jamel Eddine Bencheikh, Les Mille et Une Nuits ou la parole prisonnière, Paris, Gallimard, 1988, p. 177-194. Remarquer que l'ange qui préside à l'alternance du jour et de la nuit, que Buluqiyâ rencontre au cours de sa pérégrination, porte, incrustées entre ses yeux, une ligne noire et une blanche, qui grandissent tour à tour aux moments voulus (L. López Baralt, op. cit., p. 58, 92, 115 sq., 143) : c'est, exactement, le motif des deux vetas de l'alcuba du conte morisque (voir supra n. 15). 
Yima ${ }^{102}$, ou du mythe cosmogonique kurde de la Perle, qui apparaît elle aussi comme la résidence secrète, perdue au sein des eaux primordiales, d'entités mystiques suscitées en elles par l'Esprit Divin (qui les y tient recluses avant de les en faire sortir lorsqu'est venu le temps de la Manifestation) ${ }^{103}$. Les analogies ne supposent pas nécessairement filiation, mais plutôt enracinement dans un substrat commun d'images investies de croyances d'origine iranienne, comme permet par ailleurs de le supposer la probable parenté de l'alcuba morisque avec le dôme paradisiaque des contes persans, coupole ultramarine, quotidiennement visitée par l'oiseau Simurgh, d'où procèdent la grappe mystique et les quatre rivières cosmiques ${ }^{104}$.

Il faudrait aussi, en aval, détailler les développements occidentaux - notamment dans certains aspects de l'iconographie médiévale et de l'imagerie alchimique ${ }^{105}$, et jusque dans la mythologie et la

102. Noter que la résidence de ces bienheureux, séparée du reste du monde par un fleuve surnaturel ou par une mer infranchissable, se distingue, comme le vara de Yima, par son caractère collectif, de l'isolement purement individuel du héros de notre conte. Quelques textes sapientiaux, notamment certaines rédactions du Kitâb âdâb al-falâsifa attribué à Hunayn ibn Ishâq (IX siècle), annexent un mythe de ce type à la légende salomonienne : on y voit le roi hébreu se faire transporter par un zéphyr dans une île peuplée de génies pleins de sagesse pour solliciter leurs aphorismes : voir Montserrat Abumalham, « Salomón y los genios », Anaquel de Estudios Arabes, III, 1992, p. 37-46 (p. 42).

103. Voir supra n. 70 : l'Esprit divin commence par susciter cette Perle au sein de l'Océan primordial et y réside lui-même seul. Plus tard il en sort et y fait apparaître des sortes de doubles de lui-même. Au moment de créer le monde spirituel, il crée Gabriel en plongeant son regard dans la Perle, puis le jette à la mer, où il subira une série d'épreuves spirituelles (sous forme de questions) dont il ne sortira vainqueur qu'à la troisième reprise ( $c f$. supra nn. 45 et 47).

104. Voir A. Bricteux, op. cit., loc. cit. : le paradis y est figuré comme une coupole juchée au sommet d'une montagne d'or qui couronne une île inaccessible située au delà de sept mers, que seul franchit, chaque matin, l'oiseau Simurgh (cf. supra nn. 52 et 83 ); la roue céleste tourne à l'intérieur de cette coupole, d'où jaillissent quatre sources divergentes. Comparer avec l'imagerie cosmologique de l'Iran préislamique et avec le cycle des récits shiites duodécimains concernant l'Ile Verte au centre de la Mer de Blancheur, résidence mystique de l'Imam Caché : voir H. Corbin, En Islam Iranien..., t. IV, p. 330, 346 sq.

105. Voir Jurgis Baltrusaïtis, Le Moyen Age fantastique. Antiquités et exotismes dans l'Art Gothique, Paris, Flammarion, 1981, p. 194-202 : l'auteur compare l'iconographie médiévale occidentale et celle du bouddhisme (d'où procède le motif de l'auréole) et montre comment, de part et d'autre, le nimbe immatériel 
littérature celtiques (dont l'héritage « oriental » reste à explorer) ${ }^{106}$ du mystérieux motif de l'homme enfermé dans un réceptacle de cristal, dont les implications sont généralement, à la fois ou tour à tour, cosmiques, mystiques et/ou eschatologiques.

Nous avons de même signalé tout ce que doit le conte morisque à la légende d'Alexandre, dont on a vu que, notamment dans les cultures d'Europe centrale et orientale, les traditions salomoniennes sont largement tributaires.

Ces croisements ne concernent pas seulement le motif de l'exploration sous-marine et de l'homme enfermé dans une bathysphère. Le cycle alexandrin fournit aussi le modèle de la confrontation du sage et du souverain. Confrontation collective dans le cas de la rencontre avec les gymnosophistes, ou individuelle dans celui du dialogue de l'empereur avec Diogène : dans ces deux configurations, dont le conte morisque semble vouloir opérer la synthèse (puisqu'il retient de la première l'idée d'une sagesse cachée ultramarine, et de la seconde le motif du solitaire confiné dans un habitacle insolite), le représentant du pouvoir temporel sort relativement humilié du débat, au cours duquel il essuie une rebuffade ou, dans le meilleur des cas, doit reconnaître les limites, purement matérielles, de son pouvoir, incapable de satisfaire les exigences spirituelles de ses interlocuteurs ${ }^{107}$.

tend progressivement à être représenté comme un disque vitreux (plus ou moins réfléchissant), puis comme une boule de verre à l'intérieur de laquelle la figure nimbée finit par être totalement incluse, comme un poisson dans un bocal. Ce type d'évolution confirme indirectement notre hypothèse de l'enracinement de ce motif dans l'ancienne mythologie iranienne de la "Gloire Lumineuse ", dont l'iconographie bouddhiste a peut-être hérité certaines de ses formes.

106. Les îles, cités, tours, montagnes de verre abondent dans les littératures celtiques et dans la «matière de Bretagne » (notamment à propos de Glastonbury), où elles sont presque toujours associées à l'au-delà : voir Alexander Haggerty Krappe, “The Glass Mountain”, Modern Language Quarterly, VIII, 1947, p. 139-145 et id., "Avallon », Speculum, XVIII, 1943, p. 303-322. Pour le motif plus particulier qui nous concerne ici voir Michel Stanesco, «Une merveille bien énigmatique : le chevalier dans un tonneau de verre », in Le Monde et l'Autre Monde, D. Hüe, C. Ferlampin-Acher éds., Orléans, Paradigme, 2002, p. 359-368.

107. Sur ce point le conte morisque est à rapprocher de l'anecdote, qui figure dans plusieurs textes arabes, relative à la rencontre d'Alexandre avec un jeune prince habitant dans un cimetière : lorsque le conquérant macédonien 
Il y aurait enfin lieu d'observer que le thème de l'absconditio maritime du sage a dû être très précocement associé à la mythologie de la dévotion filiale, dont on a évoqué plus haut les racines indiennes : on sait que ce type de pietas est traditionnellement incarné et symbolisé, selon les Bestiaires, par la cigogne, dont on ne cesse de raconter, depuis l'Antiquité, que ses petits prennent le plus grand soin de leurs parents quand ils sont atteints par la vieillesse (et vont jusqu'à les couver pour renouveler leur plumage et les rajeunir), ce qui, selon Elien, vaut aux plus dévoués de finir leur vie sous forme humaine dans des îles océaniques dont les dieux ont voulu faire le conservatoire de la piété et de la grandeur spirituelle ${ }^{108}$.

2) N'étant réductible, en dernière analyse, à aucune des multiples légendes avec lesquelles il a cependant de notables analogies (les démons plongeurs, l'exploration sous-marine d'Alexandre, les génies embouteillés, le conte de Buluqiyâ etc.), le conte qui fait l'objet de cette étude apparaît comme une sorte de carrefour de traditions : il emprunte des motifs spécifiques à des « sources » diverses dont il subvertit par ailleurs les structures narratives et les significations, et sa généalogie n'est manifestement pas unilinéaire.

Il n'est pourtant nullement incohérent : c'est toujours au même niveau, sur la base d'une implicite référence commune à un archétype relativement stable et constant, qu'il recoupe le corpus apparemment bigarré des traditions dont il se nourrit. Ces dernières sont, comme on l'a montré, répétitivement traversées par un leitmotiv, un véritable « mythème » qui, au-delà ou en deçà de leurs spécificités

lui propose de se joindre à lui et de voir ressuscitée sa gloire et celle de ses ancêtres, le jeune homme décline l'invitation et déclare vouloir continuer à méditer sur la vanité des grandeurs, l'incomplétude de la vie et la nécessité de la prière (R. Basset, Mille et un contes, récits et légendes arabes, ed. A. Chraïbi, t. 2, p. 278-279, «Alexandre et le fils d'un roi »). On se souviendra que, dans l'Itinéraire de Zosime, les Réchabites opposent un ferme refus aux instances du souverain, qui veut les obliger à revenir à une vie normale.

108. Claudius Aelianus, De natura animalium, II, 23. Le même symbolisme est aussi associé à la huppe, oiseau salomonien par excellence, dont on connaît le rôle dans les traditions du soufisme, et à propos duquel Elien rapporte une histoire de piété filiale extrême qu'il dit d'origine indienne (ibid., XVI, 5). 
respectives et de leurs origines hétérogènes, leur confère une certaine solidarité réticulaire.

Selon ce mythème récurrent - dont on a vu qu'il est souvent balisé par des allusions plus ou moins indirectes au personnage d'Al Khadir - le milieu marin est, malgré son opacité a priori hostile, habité, visité, ou simplement surveillé, par une entité mystérieuse, voire mystique, qui détient - ou en qui se condense - le principe immanent, généralement caché, d'une Sagesse radicalement autre, qui est tour à tour ou tout à la fois un savoir, un pouvoir (éventuellement une souveraineté), une justice (relevant de la théodicée ou de la maîtrise de Vérité) et une source de Vie (parfois d'immortalité). À cette Sagesse, qui est aussi lumière, le héros humain aspirant à l'une ou l'autre forme de souveraineté doit se confronter, la rencontre pouvant apparaître comme une compétition, une ordalie, un entretien sapiential, une communion mystique, et pouvant aboutir à une apothéose, un échec ou une simple fin de non recevoir... Ces « expériences sapientiales » ne sont nullement abstraites : susceptibles d'être projetées sur des personnages, elles recoupent des structures narratives et alimentent un certain « folklore », dont les connotations philosophiques ou mystiques n'entravent en rien le fonctionnement traditionnel.

Le personnage d'Al Khadir ne serait, dans cette perspective, que la version islamisée de cette entité mythique qui, dans d'autres contextes, peut apparaître sous les traits mobiles des «Vieux de la Mer » grecs (Protée, Nérée, Glaucos etc.), des « Descendants des Eaux » indoeuropéens (Apam Napat) ${ }^{109}$, et, dans une moindre mesure, encore à déterminer, du «Survivant du Déluge » sémitique, qui parvient à préserver, contre vents et marée, l'héritage civilisateur des sages antédiluviens, mi humains mi ichthyomorphes.

Ces personnages, qui sont souvent dotés d'un statut intermédiaire entre l'humain et le divin, résident aux confins de l'univers, ou dans des îles lointaines et apparaissent, de par ce privilège d'extra-

109. Voir supra nn. 39 et 67. Voir également Claude Sterckx, Les dieux protéens des Celtes et des Indo-Européens, Bruxelles, Mémoires de la Soc. Belge d'Études Celtiques, 1994. 
territorialité, ou du fait de leur nature protéenne, largement insaisissables et inaccessibles ${ }^{110}$. Ils sont en rapport intime avec l'élément aquatique, généralement marin, et occasionnellement avec le monde des poissons. Ils détiennent enfin une Vérité cachée, de type oraculaire, d'allure énigmatique ou paradoxale ${ }^{111}$, ou, plus concrètement, des substances (ou talismans) dont l'obtention confère au héros humain, qui a su les conquérir ou se les faire donner, une forme de souveraineté ou de super-vie. Cette obtention n'intervient généralement qu' au terme d'un processus « initiatique », voire mystagogique, qui implique le franchissement des limites de l'ordre mondain et l'accès à un mode d'appréhension divinatoire. Les indéchiffrables actions commises par le «Serviteur de Dieu », compagnon de voyage de Moïse, dans la légende coranique (qui ne serait autre, selon les commentateurs, que le Verdoyant Khadir), transposent dans les termes de la théodicée les énigmatiques éclats de rire des poissons, l'hilarité intempestive d'Asmodée ou de l'homme sauvage des contes merveilleux juifs et arabes ${ }^{112}$, manifestations qui sont toutes liées à la détention d'un savoir caché inaccessible au commun des mortels : pour obtenir ce savoir il faut s'en remettre au bon vouloir de ces prophètes, ou les contraindre à le délivrer, ou encore accomplir sous leur direction un parcours initiatique et sapiential qui suppose l'affranchissement des conditionnements de la conscience commune.

3) Le conte morisque n'explicite pas ce message mystérique en tant que tel : il se contente de le mettre en images et en situation. Il ne met pas directement en scène un personnage du genre d'Al Khadir, dont la présence latente n'est perçue qu'allusivement, et

110. Les Réchabites et autres Gymnosophistes ultramarins leur doivent probablement quelque part de leur relative incommunicabilité, et la tradition littéraire des dialogues sapientiaux avec ces sages semi-paradisiaques s'entrecroise avec l'inépuisable mythologie des captures d'hommes sauvages-prophètes (séduits, enivrés ou habilement saisis alors qu'ils sont en pleine transformation) qu'il faut « enserrer» avant de les forcer à dévoiler leurs savoirs cachés.

111. Voir Marcel Detienne, Les Maîtres de Vérité dans la Grèce archaïque, Paris, F. Maspéro, 1967, p. 29-50 (« Le Vieux de la Mer»).

112. C. Brémond, « Pourquoi le poisson a ri », Poétique, XLV, 1981, p. 9-19. Voir également A. H. Krappe, «Le Rire du Prophète», in Studies in English Philology (= Mélanges F. Klaeber), Minneapolis, 1929, p. 340-361. 
par l'intermédiaire de son protégé et lieu-tenant qu'est ici le jeune homme reclus dans un vert habitacle de verre.

Le récit se contente de remettre en perspective le modèle sapiential salomonien en le confrontant à une variation atypique sur le thème para-hagiologique de l'«esseulé »113. À l'afrâd cher à Ibn Arabi, représentant du degré supérieur de la sainteté qu'est la «station de la proximité », notre jeune homme encapsulé ressemble fort, en ce que Dieu a pris soin de le soustraire au monde et de le cacher : ne participant en rien aux miracles dont il est passivement et discrètement l'objet, apparemment abandonné à l'incessante et opaque agitation des flots, jeté au milieu de la guerre éternelle des poissons, au sein desquelles il demeure imperturbé, à peine concerné par l'alternance rythmique du diurne et du nocturne, il ne formule aucune sentence, ne délivre aucun sermon, ne suggère aucun projet spirituel. Son exemplarité, quoique évidente, est totalement implicite. On connaît, par Ibn Arabî, l'affinité de ces « esseulés » avec Al Khadir, qui est en quelque sorte leur patron, et avec l'élément aquatique, plus particulièrement avec ces mythiques hommes sous-marins qui, tel l' « Abdallah de la Mer» des Mille et une nuits, adorent la divinité au fond des abysses ${ }^{114}$.

Il n'est pas étonnant que les Morisques, ces étrangers sur leur propre terre, ces exilés en puissance, se soient reconnus dans des figures de ce type, quitte à les réinventer ou réinterpréter pour les conformer à leur propre situation existentielle. Ils ont dû y voir l'expression d'une nostalgie de leurs racines orientales, la projection de la nécessité stratégique d'une occultation de leurs véritables références spirituelles, en même temps que la manifestation de leurs attentes eschatologiques.

L'étrange histoire du jeune homme reclus en pleine mer des ténèbres au sein d'une protectrice alcuba de verre n'a pu être perçue par eux que comme l'image allégorique de leur propre isolement au

113. Voir Michel Chodkiewicz, Le Sceau des Saints. Prophétie et Sainteté dans la doctrine d'Ibn Arabî, Paris, Gallimard, 1986, p. 133 sq., 175, et Ibn Arabî, Les illuminations de La Mecque (textes choisis), M. Chodkiewicz éd., Paris, Sindbad, 1988, p. 65-67, 332-347, 393, 525 sq., 571-573, 576 sq.

114. H. Toelle, op. cit., p. 334 sq. 
sein d'une société hostile. La confrontation avec un roi présomptueux, et finalement humilié, de ce jeune représentant d'une sagesse élémentaire et discrète, héraut de cette piété filiale qui est le seul refuge d'un peuple menacé dans la continuité de son existence culturelle, ne pouvait être interprétée, à leurs yeux, que comme la métaphore de leur rapport à un pouvoir royal tyrannique ${ }^{115}$. Un pouvoir contre lequel, en attendant l'expulsion ultramarine, il ne restait d'autre refuge symbolique que celui d'une fragile, maternelle et mystique prison de cristal.

15 rue du Faubourg Saint-Antoine 75011 Paris

115. M. J. Fernández Fernández, « Análisis formal y comparado de un relato aljamiado morisco », Al Qantara, IX, 1988, p. 101-119 (représentation négative de la royauté dans un conte du Libro de los Castigos). 



\section{Appendice \\ Salomon et le jeune homme dans la coupole de verre}

On raconte qu'un jour Salomon eut envie de savoir quelle est la profondeur de la mer et ce qu'on y peut trouver. Il choisit cent démons parmi les plus habiles ; sur les cent il en retint cinquante, sur les cinquante vingt-cinq, sur les vingt-cinq douze, sur les douze six et sur les six trois.

À l'un d'entre eux il ordonna d'aller visiter les mers, d'observer ce qui s'y trouve et ce qu'y font les créatures marines. Ayant parcouru toutes les mers, le démon n'y vit que des poissons, dont les plus gros dévoraient les plus petits. Il revint rendre compte de sa mission au roi Salomon fils de David - alahi çalam (la paix soit sur lui) - et lui dit ce qu'il avait vu dans la mer.

Le roi dépêcha un deuxième démon, avec l'ordre de sonder le fond de la mer. Le démon partit mais ne put nullement atteindre le fond. En chemin il rencontra dans la mer un ange qui lui dit :

- « Où vas-tu, démon maudit ?».

Le démon répondit :

- «Salomon m'a ordonné de sonder le fond de la mer, mais je n'ai pu l'atteindre ».

L'ange dit :

- «Comment aurais-tu pu l'atteindre, alors que personne d'autre que Dieu n'en a connaissance ? Il y a cent ans de cela, j'ai moi-même, en ce même endroit, vu un marin qui réparait sur son navire un madrier brisé par la tempête : son outil lui échappa et tomba à l'eau. Or cet outil n'a toujours pas atteint le fond !».

Il dit et s'en alla. Continuant son voyage maritime, le démon finit par tomber sur une coupole de verre verte. Elle était attaquée par les ondes et les poissons, qui ne lui causaient néanmoins aucun dommage. Il revint raconter au roi Salomon ce que lui avait dit l'ange et l'informa de sa découverte de la coupole de verre verte. Apprenant cela, Salomon fit convoquer ses cent démons et leur ordonna d'aller chercher en mer ladite coupole verte et de la lui apporter. 
L'ordre fut exécuté et les démons déposèrent la coupole aux pieds du roi Salomon. Or à l'intérieur de la coupole se trouvait un jeune homme vêtu de blanc. Le roi s'adressa à lui :

- « Jeune homme, pour quelle raison te trouvais-tu dans cette coupole de verre verte? $»$.

Le jeune homme répondit :

- « Oh roi, tu sauras que mes parents étaient très âgés : je les ai soignés et servis comme ils l'avaient fait pour moi quand j'étais enfant, je les ai lavés et maintenus propres comme des bébés. Mon père est mort le premier. Quand ma mère fut à son tour sur le point de mourir, elle pria Dieu de me soustraire au monde, afin qu'après sa disparition je ne sois pas corrompu par les désirs et les vices d'ici bas et ne perde pas le capital de mérites que j'avais gagné. Dieu exauça la prière de ma mère : deux anges vinrent me prendre en Son Nom et ils me déposèrent en pleine mer dans cette coupole de verre verte ».

Le roi dit :

- « Depuis combien de temps t'y trouves-tu ?».

Le jeune homme répondit :

- « Depuis vingt-six ans ».

Le roi demanda :

- «Comment te nourris-tu ? Comment te désaltères-tu ?».

Il répondit :

- « Tous les matins un oiseau blanc m'apporte dans son bec une substance blanche qui me sert d'aliment et de boisson ».

Le roi dit :

- « Jeune homme, comment distingues-tu le jour de la nuit ?».

Il répondit :

- « Vois-tu, oh roi, cette rainure blanche ? Quand sa blancheur s'intensifie, je sais que c'est le jour ».

Le roi dit :

- «Comment sais-tu que c'est la nuit?».

Le jeune homme répondit :

- «Quand cette rainure noire s'agrandit, je sais que c'est la nuit ».

Le roi demanda :

- « Oh jeune homme : veux-tu rester ici avec moi ?».

Le garçon répondit :

- «Non, je te demande de me faire remettre où j'étais, afin que s'accomplisse ce que Dieu a disposé à mon sujet ».

Et le jeune homme réintégra sa coupole, puis en referma la porte de l'intérieur. Les démons l'emportèrent et le remirent en mer, là même où ils l'avaient trouvé. 


\section{Çulaiman y el mancebo de la alcuba de vidrio}

Fue recontado que a Çulaiman le vino gana de llegar a saber donde llegaba la fondera del mar i lo que había en él, y escogió de los assaytanes çiento de los más avisados ; i de ciento, cincuenta ; y de cincuenta, vinticinco, de los vinticinco doce ; y de los doce seis ; y de los seis tres.

I mandó al uno que fuese i mirase las mares, i lo que había en ellas, i lo que fazía en ellas los halecados della. I fue i andó todas las mares, i no vio sino peçcados en ella que se comían los grandes a los chicos. I vino i dio razón dello al rey Çulaiman, ficho de Dawud, alahi çalam, i dixole lo que había visto en los mares.

I mandó al segundo de los assaytanes, i dixole que fuese i mirase adónde llegaba lo más fondo de las mares. I fue i no pudo llegar a los fondos de la mar en ninguna manera. I andando por la mar, encontró un almalaque, i dixole :

- « ¿ Adónde vas ? ; ya Ebliç, maldito ! ».

I dixole :

- « Ame mandalo Çulaiman que le supiese adónde llegaba lo más fondo de los mares i no é podido llegar a ello. »

I díxole el almalaque :

- « ¿ Cómo has de llegar tú a ello ni nadie sino el saber de Allah, mi señor ? Que yo a cien años que, estando yo en este lugar, estaba un marinero apañando su navío que se le había quebrado una madera de un golpe de la mar, i se le cayó el açuela de su mano, i no á llegado aún al suelo de lo fondo de la mar. »

I con aquello se departío dél Ebliç. I, andando por la mar topó en él, una alcuba de vidrio verde. I vido que la combatían las ondas y los peces de la mar, i no le fazían nocimiento alguno. I vino al rey Çulaiman i contóle lo que le había dicho el almalaque, i cómo había visto aquella alcuba verde de vidrio en la mar.

I cuando oyó Çulaiman aquello mandó todos los cien aljinnes delante dél, i mandóles que fuesen i le trajesen aquella alcuba verde de la mar ansí como estaba.

I fueron a la mar, i tomaron el alcuba verde, i traséronla delante del rey Çulaiman ; i había dentro del alcuba un mançebo vestido de blanco, i díxole el rey :

- « ¡ Ya mancebo ! ¿Qué fue la causa de tu estar en esta alcuba de vidrio verde ?»

Dixole el mancebo :

- « i Ya rey! Tú as de saber que yo tenía a mi padre y a mi madre muy viechos, i yo les fize el tratamiento i serviçio que ellos me fizieron a mí de niño, i los lavaba i alimpiaba como a las criaturas. I murió mi padre primero que mi madre.I cuando vino a morir mi madre ruegó ada Allah que me sacase del mundo, en muriendo ella, porque no me alcançasen los apetitos i viçios del mundo para que no pirdiese aquel gualardón que tenía. I oyó Allah la rogaría de mi madre, i vinieron dos almalaques, i tomáronme con el poder de Allah, i pusiéronme en la mar, en esta alcuba de vidrio verde. »

I díxole el rey :

- « ¿ I cuánto tiempo a que estás en ella ?» 
Dixo el mancebo :

- « Tiempo a de vinte i seis años. »

Díxole el rey :

- « ¿ I qué es tu comer i tu beber?»

Dixo :

- «Viéneme cada mañana un ave blanca i tráeme en su pico una cosa blanca, i aquello me sustenta de comer i de beber cada día. »

I dixóle el rey :

- « ¡ Ya mancebo ! ¿ Cómo ves cuando es de día o de noche ?»

Dixo :

- « i Ya rey !, ¿ Ves esta veta blanca ? Cuando ella creçe en blancura, sé que es de día. »

Dixo el rey :

- « ¿ Cómo sabes ques de noche?»

Dixo el mancebo :

- « Cuando esta veta negra crece, laora sé que es de noche. »

Dixole el rey :

- « ¡ Ya mançebo !, ¿ Quieres estarte aquí conmigo ?»

Dixo el mançebo :

- « No, sino que me tornes adonde estaba i cumpla Allah su chuzgo sobre mi .»

Y dentróse el mançebo en su alcuba, i çerró la puerta por de dentro. I tomáronlo los asaitanes, i levaronlo a la mar a donde mesmo estaba.

(Transcription de M. J. Fernández Fernández, reproduite dans Y. Cardaillac Hermosilla, La Magie en Espagne: Morisques et Vieux Chrétiens aux XVI et XVII siècles, Zaghouan, Fondation Temimi, 1996, p. 226 sq.) 